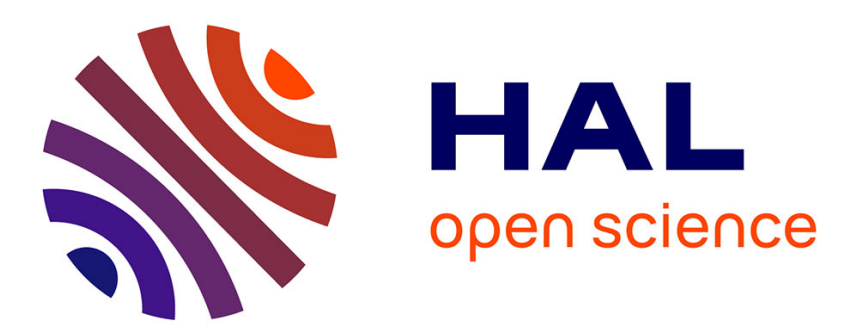

\title{
Gâteaux type path-dependent PDEs and BSDEs with Gaussian forward processes
}

\author{
Adrien Barrasso, Francesco Russo
}

\section{To cite this version:}

Adrien Barrasso, Francesco Russo. Gâteaux type path-dependent PDEs and BSDEs with Gaussian forward processes. Stochastics and Dynamics, 2022, 22, pp.2250007,. 10.1142/S0219493722500071. hal-02197479

\section{HAL Id: hal-02197479 \\ https://hal.science/hal-02197479}

Submitted on 30 Jul 2019

HAL is a multi-disciplinary open access archive for the deposit and dissemination of scientific research documents, whether they are published or not. The documents may come from teaching and research institutions in France or abroad, or from public or private research centers.
L'archive ouverte pluridisciplinaire HAL, est destinée au dépôt et à la diffusion de documents scientifiques de niveau recherche, publiés ou non, émanant des établissements d'enseignement et de recherche français ou étrangers, des laboratoires publics ou privés. 


\title{
Gâteaux type path-dependent PDEs and BSDEs with Gaussian forward processes
}

\author{
Adrien BARRASSO * $\quad$ Francesco RUSSO ${ }^{\dagger}$
}

June 26th 2019

\begin{abstract}
We are interested in path-dependent semilinear PDEs, where the derivatives are of Gâteaux type in specific directions $k$ and $b$, being the kernel functions of a Volterra Gaussian process $X$. Under some conditions on $k, b$ and the coefficients of the PDE, we prove existence and uniqueness of a decoupled mild solution, a notion introduced in a previous paper by the authors. We also show that the solution of the PDE can be represented through BSDEs where the forward (underlying) process is $X$.
\end{abstract}

MSC 2010 Classification. 60G15; 60H30; 35S05; 60J35; 45D05

KEY WORDS AND PHRASES. Gaussian processes; Volterra processes; path-dependent PDEs; decoupled mild solutions; BSDEs.

\section{Introduction}

Backward SDEs (in short BSDEs) are naturally linked to non-linear deterministic evolution equations. In one of their pioneering work [22], Pardoux and Peng showed that Markovian BSDEs for which the randomness comes from an underlying which is the solution of a classical SDE, are linked to classical semilinear PDEs. In this framework an impressive amount of papers has been produced.

In the recent times, particular attention was devoted to the case when the driver and terminal condition of the BSDE depend on the whole path of the forward underlying process which can be a Brownian motion. Those are of type

$$
Y^{s, \eta}=\xi\left(\left(B_{t}^{s, \eta}\right)_{t \in[0, T]}\right)+\int_{.}^{T} f\left(r,\left(B_{t}^{s, \eta}\right)_{t \in[0, r]}, Y_{r}^{s, \eta}, Z_{r}^{s, \eta}\right) d r-\int_{.}^{T} Z_{r}^{s, \eta} d B_{r}^{s, \eta}
$$

*ENSTA Paris and Ecole Polytechnique, Institut Polytechnique de Paris 828. E-mail: adrien.barrasso@polytechnique.edu

†ENSTA Paris, Institut Polytechnique de Paris, Unité de Mathématiques appliquées, 828, boulevard des Maréchaux, F-91120 Palaiseau, France. E-mail: francesco.russo@ensta-paris.fr 
where $B$ is a Brownian motion and for any $s \in[0, T], \eta \in \mathbb{D}\left([0, T], \mathbb{R}^{d}\right), B^{s, \eta}=$ $\eta(\cdot \wedge s)+\left(B \cdot \vee s-B_{s}\right)$. If in the Markovian case those were related to usual PDEs, in the present path-dependent framework, those were linked to the so called path-dependent PDEs (see for instance $[23,13]$ ) of the form

$$
\left\{\begin{array}{l}
D \Phi+\frac{1}{2} \operatorname{Tr}\left(\nabla^{2} \Phi\right)+f(\cdot, \cdot, \Phi, \nabla \Phi)=0 \quad \text { on }[0, T] \times \mathbb{D}\left([0, T], \mathbb{R}^{d}\right) \\
\Phi_{T}=\xi
\end{array}\right.
$$

There, $D$ (resp. $\nabla$ ) is the horizontal (resp. vertical) derivative introduced in [12]. For (1.2) the authors discussed classical or viscosity (probabilistic) type solution. Variants of it, replacing the Brownian motion with the solution of path-dependent SDEs were considered for instance by $[8,7]$ with a different formalism. [7] for instance introduced the notion of stong-viscosity solution (based on approximation techniques), which constitutes a purely analytic concept.

Indeed such path-dependent PDEs have been investigated by several methods. For instance strict (classical, regular) solutions have been studied in $[11,15,8]$ under the point of view of Banach space valued stochastic processes. Another interesting approach (probabilistic) but still based on approximation (discretizations) was given by [21]. More recently, [5] produced a viscosity solution to a more general path-dependent (possibly integro)-PDE through dynamic risk measures. In all those cases the solution $\Phi$ of (1.2) was associated to the component $Y^{s, \eta}$ of the solution couple $\left(Y^{s, \eta}, Z^{s, \eta}\right)$ of (1.1) with initial time $s$ and initial condition $\eta$. A challenging link to be explored was the link between $Z^{s, \eta}$ and the solution of the path-dependent PDE $\Phi$. For instance in the case of Fréchet $C^{0,1}$ solutions $\Phi$ defined on $C([0, T])$, then $Z^{s, \eta}$ is equal to the "vertical" derivative $\nabla \Phi$, see for instance [16].

An important step forward concerning path-dependent PDEs associated with BSDEs involving a solution of a path-dependent SDEs including the possibility of jumps and coefficients which were not necessarily continuous was done in [2]. The concept of solution was there the decoupled mild solution which is based on semigroup type techniques. That notion, is competitive with the notion of viscosity solution, especially when such viscosity solutions do not necessarily exist. Moreover, that notion of solution also provides a solution to the so called identification problem, meaning that it links the second component $Z$ of the BSDE, to the PDE.

The natural question raised by this paper is the following. What about the case when the Brownian motion $B$ is replaced with a (non-Markovian, nonsemimartingale) process such as fractional Brownian motion? The idea is to extend the consideration of [2] to this framework. The basic reference paper for this work is [26], that considered for the first time a BSDE which forward process was the solution of a Volterra SDE. This includes the kind of Gaussian processes which we consider. They related this BSDE to a Gâteaux type PDE close to (1.6) by showing that if the PDE admits a classical solution, that solution provides a solution of the BSDE. Our work provides the converse implication. We start from the well-posedness of a class of BSDEs, and show that they produce, under very mild regularity assumptions on the coefficients, a decoupled mild solution to the path-dependent PDE. 
Let $(\Omega, \mathcal{F})$ be the canonical space where $\Omega$ is the set $\mathcal{C}_{0}\left([0, T], \mathbb{R}^{d}\right), d \geq 1$, of $\mathbb{R}^{d}$-valued continuous functions on $[0, T]$ vanishing at 0 , equipped with its uniform norm and $\mathcal{F}$ its Borel $\sigma$-field.

We fix $b:[0, T] \times[0, T] \rightarrow \mathbb{R}^{d}$ and $k:[0, T] \times[0, T] \rightarrow \mathcal{M}_{d}(\mathbb{R})$ some two parameters functions such that for all $t \in[0, T], b(\cdot, t), k(\cdot, t)$ vanish on $[0, t[$ and are continuous, admitting a right-derivative on $[t, T]$.

On the canonical space, we consider a Gaussian measure $\mathbb{Q}$ under which there exists a $d$-dimensional Brownian motion $B$ such that the canonical process $X$ admits the representation

$$
X_{t}=\int_{0}^{t} b(t, r) d r+\int_{0}^{t} k(t, r) d B_{r}, \quad t \in[0, T] .
$$

For every "initial time and path" $(s, \eta)$ we introduce the law $\mathbb{Q}^{s, \eta}$ of $X$ conditioned by the fact that, on $[0, s], X$ coincides with the path $\eta$. For every $(s, \eta)$, $\mathbb{Q}^{s, \eta}$ is a Gaussian measure of mean function $m_{s}[\eta]$, where $m_{s}$ is a continuous linear operator on $\Omega$. The reader can refer to [6] concerning Gaussian measures and related notions, see also Definition 3.2.

We will show that $\left(\mathbb{Q}^{s, \eta}\right)_{(s, \eta) \in[0, T] \times \Omega}$ defines what we call a path-dependent canonical class, see Definition 2.4, notion which was introduced by the authors in [4]. This concept extends the well-known historical notion of Markov canonical class to the path-dependent (therefore non-Markovian) setting.

Given this set of probability measures, under every $\mathbb{Q}^{s, \eta}$, we consider the BSDE (indexed by $(s, \eta)$ )

$$
Y_{\cdot}^{s, \eta}=\xi(X)+\int_{.}^{T} f\left(r, X, Y_{r}^{s, \eta}, \frac{d\left\langle M^{s, \eta}, m^{T, s, \eta}\right\rangle_{r}}{d r}\right) d r-\left(M_{T}^{s, \eta}-M_{\cdot}^{s, \eta}\right),
$$

where $m^{T, s, \eta}: t \longmapsto \mathbb{E}^{s, \eta}\left[X_{T} \mid \mathcal{F}_{t}\right]$ is the driving martingale of the BSDE. In the case when $k(t, \cdot) \equiv \mathbb{1}_{[0, t]}$ and $b \equiv 0$ then this driving martingale $m^{T, s, \eta}$ is $\mathbb{P}^{s, \eta}$-a.s. equal to $X$ and is the conditioned Brownian motion $B^{s, \eta}$ appearing in (1.1). This case was already considered in a more general framework, in [2]. The main aim of this paper is to study the path-dependent PDE which replaces (1.2) when one considers the previous BSDE (1.4) instead of (1.1).

Thanks to the theory which we have developed in [4], we can associate to the family of probability measures $\left(\mathbb{Q}^{s, \eta}\right)_{(s, \eta) \in[0, T] \times \Omega}$ what we call a pathdependent system of projectors $\left(P_{s}\right)_{s \in[0, T]}$, a notion which replaces the one of Markovian semigroup. We define the linear operator $\tilde{A}$, acting on a domain $\mathcal{D}(\tilde{A})$ of functions $\tilde{\Phi}$ defined on $\left.\mathbb{D}\left([0, T], \mathbb{R}^{d}\right)\right)$ by

$$
\tilde{A}(\tilde{\Phi})_{t}:=D \tilde{\Phi}_{t}+\nabla_{b(\cdot, t)} \tilde{\Phi}_{t}+\frac{1}{2} \sum_{i \leq d} \nabla_{k_{i}(\cdot, t)}^{2} \tilde{\Phi}_{t}, \quad t \in[0, T] .
$$

In (1.5), $\nabla_{h}$ (resp. $\nabla_{h, \ell}^{2}$ ) is the first (resp. second) order Gâteaux type derivatives in the direction $h$ (resp. $h, \ell$ ) and $D$ is a time derivative. Those operators act on functionals defined on a set of cadlag functions. Again when 
$k(t, \cdot) \equiv \mathbb{1}_{[0, t]}$ and $b \equiv 0$ and if $\Phi_{t}(\omega)=\tilde{\Phi}_{t}\left(\omega^{t}\right)$, then $\nabla_{k(\cdot, t)} \tilde{\Phi}_{t}\left(\omega^{t}\right)=\nabla \Phi_{t}(\omega)$, where $\nabla$ is now the vertical derivative introduced in [12].

We introduce the mean random field $m=\left(m_{s}\right)_{s \in[0, T]}$, where $m_{s}[\eta](t)$ is the conditional expectation of $X_{t}$ knowing that $X$ coincides with $\eta$ on $[0, s]$, see Proposition 3.7. In particular $m^{T}:(s, \eta) \longmapsto m_{s}[\eta](T)=\mathbb{E}^{s, \eta}\left[X_{T}\right]$ is called prediction martingale in the literature, see for instance Remark 3.2 in [24].

Then we introduce the operator $A$ on a certain domain $\mathcal{D}(A)$ which to each $\Phi=\tilde{\Phi} \circ m$ associates $(\tilde{A}(\tilde{\Phi})) \circ m$. We also introduce in Definition 4.15, the bilinear operator $\Gamma$ which to any $\Phi, \Psi \in \mathcal{D}(A)$ maps $A(\Phi \Psi)-\Phi A(\Psi)-\Psi A(\Phi)$. This operator was already introduced in another context in [2] and extends the carré du champ operator appearing in the Markov processes literature, see [10] for instance.

We show in Proposition 4.12 that $A$ is a weak generator of $\left(P_{s}\right)_{s \in[0, T]}$, see Definition 2.15. That operator $A$ is therefore linked to the probability measures $\left(\mathbb{Q}^{s, \eta}\right)_{(s, \eta) \in[0, T] \times \Omega}$ mentioned above, and this will lead us to show that the BSDE (1.4) permits to solve the following semilinear path-dependent PDE which we denote $P D P D E(f, \xi)$ :

$$
\left\{\begin{array}{l}
A(\Phi)+f\left(\cdot, \cdot, \Phi, \Gamma\left(m^{T}, \Phi\right)\right)=0 \text { on }[0, T] \times \Omega \\
\Phi_{T}=\xi, \text { on }[0, T] \times \Omega .
\end{array}\right.
$$

A process $Y$ will be called a decoupled mild solution of $P D P D E(f, \xi)$ if there exists an $\mathbb{R}^{d}$-valued auxiliary process $Z$ such that for all $(s, \eta) \in[0, T] \times \Omega$

$$
\left\{\begin{aligned}
Y_{s}(\eta) & =P_{s}[\xi](\eta)+\int_{s}^{T} P_{s}\left[f\left(r, \cdot, Y_{r}, Z_{r}\right)\right](\eta) d r \\
\left(Y m^{T}\right)_{s}(\eta) & =P_{s}\left[\xi X_{T}\right](\eta)-\int_{s}^{T} P_{s}\left[\left(Z_{r}-m_{r}^{T} f\left(r, \cdot, Y_{r}, Z_{r}\right)\right)\right](\eta) d r .
\end{aligned}\right.
$$

We emphasize that decoupled mild solutions were introduced in the framework of classical parabolic PDEs in [3], and in the path-dependent framework in [2]. Those extend the notion of classical solution i.e. a functional $\Phi$ in the domain $\mathcal{D}(A)$ fulfilling (1.6).

The main result of this paper is Theorem 4.28 which shows that when $\xi$ is measurable with polynomial growth and $f$ is measurable with polynomial growth in $\omega$ and uniformly Lipschitz in the last two variables, then $P D P D E(f, \xi)$ admits a unique decoupled mild solution $Y$.

As anticipated, another feature of the paper is that the solution admits a probabilistic representation. Indeed, the unique decoupled mild solution of $P D P D E(f, \xi)$ is given by

$$
Y:(s, \eta) \longmapsto Y_{s}^{s, \eta},
$$

where $Y^{s, \eta}$ is the solution of $\operatorname{BSDE}(1.4)$.

When $b \equiv 0$ and $k(t, \cdot) \equiv \mathbb{1}_{[0, t]}$ for all $t$, then for every $(s, \eta), \mathbb{Q}^{s, \eta}$ is the law of the "conditioned" Brownian motion $B^{s, \eta}$ introduced after (1.1). In this case, our BSDE (1.4) is simply (1.1) and (1.6) becomes (1.2). Existence and uniqueness of a decoupled mild solution in this case was already shown in our previous paper [2]. That paper includes the case of (semimartingale-)solutions 
to path-dependent SDEs with jumps; in that case the driving martingale of the BSDE is the martingale component of the semimartingale $X$.

The paper is organized as follows. Section 2 recalls some notions and results related to path-dependent canonical classes and systems of projectors, which were introduced by the authors in [4]. Section 3 is mainly devoted to showing that, under some conditions, a Gaussian measure induces a path-dependent canonical class when considering its regular conditional probability distributions, see Proposition 3.10 for the centered case and Proposition 3.22 for the case with a drift. Section 4 is the main section of the paper. It introduces the path-dependent PDE for which we will show well-posedness, and the associated BSDE. First, Section 4.1 introduces the assumptions on $k, b$, see Hypothesis 4.1. Then in Section 4.2 we define the linear operators $\tilde{A}$ and $A$ appearing in (1.5) and (1.6) with the corresponding domains, see Definition 4.8. Theorem 4.11 provides an Itô formula for elements of $\mathcal{D}(A)$. In Section 4.3 we introduce the driving martingale of the BSDE (see Notation 4.19) and study its properties, see Proposition 4.20. Finally, in Section 4.4, we consider the path-dependent PDE (4.10) and show in Theorem 4.28 that, under Hypothesis 4.22, it admits a unique decoupled mild solution and a probabilistic representation through the BSDE (4.13). Proposition 4.29 shows that any classical solution of (4.10) is also a decoupled mild solution, and conversely that if the unique decoupled mild solution belongs to $\mathcal{D}(A)$ then it is quasi surely (see Definition 4.18) a classical solution.

\section{Preliminaries, path-dependent canonical classes and systems of projectors}

In this paper, we will make use of notions and results concerning path-dependent canonical classes, which were introduced in Section 3 of[4]. We give here the main definitions and results related to that concept.

We start by fixing some basic vocabulary and notations.

Notation 2.1. A topological space $E$ will always be considered as a measurable space equipped with its Borel $\sigma$-field which shall be denoted $\mathcal{B}(E)$.

Let $(\Omega, \mathcal{F}),(E, \mathcal{E})$ be two measurable spaces. A measurable mapping from $(\Omega, \mathcal{F})$ to $(E, \mathcal{E})$ shall often be called a random variable (with values in $E$ ), or in short r.v.

Given a measurable space $(\Omega, \mathcal{F})$, for any $p \geq 1$, the set of real valued random variables with finite $p$-th moment under probability $\mathbb{P}$ will be denoted $\mathcal{L}^{p}(\mathbb{P})$ or $\mathcal{L}^{p}$ if there can be no ambiguity concerning the underlying probability.

Given a stochastic basis, for any cadlag locally square integrable martingales $M, N$, we denote $\langle M, N\rangle$ (or simply $\langle M\rangle$ if $M=N$ ) their (predictable) angular bracket.

Notation 2.2. We fix $T \in \mathbb{R}_{+}^{*}$ and $d \in \mathbb{N}^{*} . \Omega:=\mathcal{C}_{0}\left([0, T], \mathbb{R}^{d}\right)$ will denote the space of continuous functions from $[0, T]$ to $\mathbb{R}^{d}$ vanishing at 0 . 
For every $t \in[0, T]$ we denote the coordinate mapping $X_{t}: \omega \mapsto \omega(t)$ and we define on $\Omega$ the $\sigma$-field $\mathcal{F}:=\sigma\left(X_{r} \mid r \in[0, T]\right)$. The coordinates of $X$ are denoted $X^{1}, \cdots, X^{d}$. On the measurable space $(\Omega, \mathcal{F})$, we introduce the initial filtration $\mathbb{F}^{o}:=\left(\mathcal{F}_{t}^{o}\right)_{t \in[0, T]}$, where $\mathcal{F}_{t}^{o}:=\sigma\left(X_{r} \mid r \in[0, t]\right)$, and the (rightcontinuous) canonical filtration $\mathbb{F}:=\left(\mathcal{F}_{t}\right)_{t \in[0, T]}$, where $\mathcal{F}_{t}:=\bigcap_{s \in] t, T]} \mathcal{F}_{s}^{o}$ if $t<T$ and $\mathcal{F}_{T}:=\mathcal{F}_{T}^{o}=\mathcal{F} .(\Omega, \mathcal{F}, \mathbb{F})$ will be called the canonical space, and $X$ the canonical process. On $[0, T] \times \Omega$, we will denote by $\mathcal{P r o}^{o}$ (resp. Pre ${ }^{o}$ ) the $\mathbb{F}^{o}$-progressive (resp. $\mathbb{F}^{o}$-predictable) $\sigma$-field. $\Omega$ will be equipped with the sup norm $\|\cdot\|_{\infty}$ which makes it a Banach space, and for which the Borel $\sigma$-field is $\mathcal{F}$.

$\mathcal{P}(\Omega)$ will denote the set of probability measures on $\Omega$ and will be equipped with the topology of weak convergence of measures which also makes it a Polish space being $\Omega$ itself Polish, see Theorems 1.7 and 3.1 in Chapter 3 of [14]. It will also be equipped with the associated Borel $\sigma$-field.

Notation 2.3. For any $\omega \in \Omega$ and $t \in[0, T]$, the path $\omega$ stopped at time $t$ $r \mapsto \omega(r \wedge t)$ will be denoted $\omega^{t}$.

Definition 2.4. A path-dependent canonical class will be a set of probability measures $\left(\mathbb{P}^{s, \eta}\right)_{(s, \eta) \in[0, T] \times \Omega}$ defined on the canonical space $(\Omega, \mathcal{F})$. It will verify the three following items.

1. For every $(s, \eta) \in[0, T] \times \Omega, \mathbb{P}^{s, \eta}\left(\omega^{s}=\eta^{s}\right)=1$;

2. for every $s \in[0, T]$ and $F \in \mathcal{F}$, the mapping

$$
\begin{aligned}
& \eta \longrightarrow \mathbb{P}^{s, \eta}(F) \\
& \Omega \longrightarrow[0,1]
\end{aligned} \text { is } \mathcal{F}_{s}^{o} \text {-measurable; }
$$

3. for every $(s, \eta) \in[0, T] \times \Omega, t \geq s$ and $F \in \mathcal{F}$,

$$
\mathbb{P}^{s, \eta}\left(F \mid \mathcal{F}_{t}^{o}\right)(\omega)=\mathbb{P}^{t, \omega}(F) \text { for } \mathbb{P}^{s, \eta} \text { almost all } \omega
$$

This implies in particular that for every $(s, \eta) \in[0, T] \times \Omega$ and $t \geq s$, then $\left(\mathbb{P}^{t, \omega}\right)_{\omega \in \Omega}$ is a regular conditional expectation of $\mathbb{P}^{s, \eta}$ by $\mathcal{F}_{t}^{o}$, see the definition above Theorem 1.1.6 in [25] for instance.

A path-dependent canonical class $\left(\mathbb{P}^{s, \eta}\right)_{(s, \eta) \in[0, T] \times \Omega}$ will be said to be progressive if for every $F \in \mathcal{F}$, the mapping $(t, \omega) \longmapsto \mathbb{P}^{t, \omega}(F)$ is $\mathbb{F}^{o}$-progressively measurable.

Remark 2.5. Given a path-dependent canonical class, one can easily show by approximation through simple functions the following. Let $Z$ be any random variable.

- For any $s \in[0, T]$ then $\eta \longmapsto \mathbb{E}^{s, \eta}[Z]$ is $\mathcal{F}_{s}^{o}$-measurable and for every $(s, \eta) \in[0, T] \times \Omega, t \geq s, \mathbb{E}^{s, \eta}\left(Z \mid \mathcal{F}_{t}^{o}\right)(\omega)=\mathbb{E}^{t, \omega}(Z)$ for $\mathbb{P}^{s, \eta}$ almost all $\omega$, provided previous expectations are finite; 
- if the path-dependent canonical class is progressive, $(t, \omega) \longmapsto \mathbb{E}^{t, \omega}[Z]$ is $\mathbb{F}^{o}$-progressively measurable, provided previous expectations are finite.

Very often path-dependent canonical classes will verify the following important hypothesis which is a reinforcement of (2.1).

Hypothesis 2.6. For every $(s, \eta) \in[0, T] \times \Omega, t \geq s$ and $F \in \mathcal{F}$,

$$
\mathbb{P}^{s, \eta}\left(F \mid \mathcal{F}_{t}\right)(\omega)=\mathbb{P}^{t, \omega}(F) \text { for } \mathbb{P}^{s, \eta} \text { almost all } \omega .
$$

Notation 2.7. $\mathcal{B}_{b}(\Omega)$ stands for the set of real bounded measurable functions on $\Omega$. Let $s \in[0, T], \mathcal{B}_{b}^{s}(\Omega)$ will denote the set of real bounded $\mathcal{F}_{s}^{o}$-measurable functions on $\Omega$. We also denote by $\mathcal{B}_{b}^{+}(\Omega)$ the subset of r.v. $\phi \in \mathcal{B}_{b}(\Omega)$ such that $\phi(\omega) \geq 0$ for all $\omega \in \Omega$.

\section{Definition 2.8.}

1. A linear map $Q: \mathcal{B}_{b}(\Omega) \rightarrow \mathcal{B}_{b}(\Omega)$ is said positivity preserving monotone if for every $\phi \in \mathcal{B}_{b}^{+}(\Omega)$ then $Q[\phi] \in \mathcal{B}_{b}^{+}(\Omega)$ and for every increasing converging (in the pointwise sense) sequence $f_{n} \underset{n}{\longrightarrow} f$ then $Q\left[f_{n}\right] \underset{n}{\longrightarrow} Q[f]$ pointwise.

2. A family $\left(P_{s}\right)_{s \in[0, T]}$ of positivity preserving monotone linear operators on $\mathcal{B}_{b}(\Omega)$ will be called a path-dependent system of projectors if it verifies the three following items.

- For all $s \in[0, T]$, the restriction of $P_{s}$ on $\mathcal{B}_{b}^{s}(\Omega)$ coincides with the identity;

- for all $s \in[0, T], P_{s}$ maps $\mathcal{B}_{b}(\Omega)$ into $\mathcal{B}_{b}^{s}(\Omega)$;

- for all $s, t \in[0, T]$ with $t \geq s, P_{s} \circ P_{t}=P_{s}$.

The proposition below states a correspondence between path-dependent canonical classes and path-dependent systems of projectors. It was the object of Corollary 3.1 of [4].

Proposition 2.9. The mapping

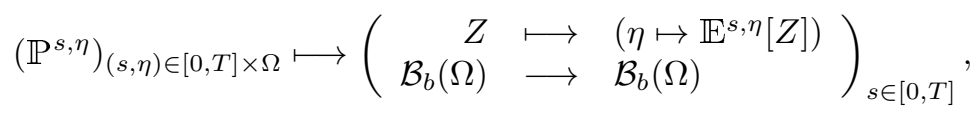

is a bijection between the set of path-dependent system of probability measures and the set of path-dependent system of projectors.

Definition 2.10. Two elements in correspondence through the previous bijection will be said to be associated.

Notation 2.11. Let $\left(P_{s}\right)_{s \in[0, T]}$ be a path-dependent system of projectors, and $\left(\mathbb{P}^{s, \eta}\right)_{(s, \eta) \in[0, T] \times \Omega}$ the associated path-dependent system of probability measures. Then for any r.v. $Z \in \mathcal{L}^{1}\left(\mathbb{P}^{s, \eta}\right), P_{s}[Z](\eta)$ will still denote the expectation of 
$Z$ under $\mathbb{P}^{s, \eta}$. In other words we extend the linear form $Z \longmapsto P_{s}[Z](\eta)$ from $\mathcal{B}_{b}(\Omega)$ to $\mathcal{L}^{1}\left(\mathbb{P}^{s, \eta}\right)$.

If $Z:=\left(Z^{1}, \cdots, Z^{d}\right)$ is an $\mathbb{R}^{d}$-valued r.v., then for all $s, P_{s}[Z]$ (if welldefined) will denote the $\mathbb{R}^{d}$-valued r.v. $\left(P_{s}\left[Z^{1}\right], \cdots, P_{s}\left[Z^{d}\right]\right)$.

For the results of this section, we are given a progressive path-dependent canonical class $\left(\mathbb{P}^{s, \eta}\right)_{(s, \eta) \in[0, T] \times \Omega}$ satisfying Hypothesis 2.6 and the corresponding path-dependent system of projectors $\left(P_{s}\right)_{s \in[0, T] \text {. }}$.

The statement below comes from Corollary 3.3 of [4].

Proposition 2.12. For every $(s, \eta) \in \mathbb{R}_{+} \times \Omega$ and $F \in \mathcal{F}_{s}, \mathbb{P}^{s, \eta}(F) \in\{0,1\}$. In particular, an $\mathcal{F}_{s}^{s, \eta}$-measurable r.v. will be $\mathbb{P}^{s, \eta}$-a.s. equal to a constant. [4].

The last notions and results of this subsection are taken from Section 5.2 of

We consider a couple $(\mathcal{D}(A), A)$ verifying the following.

\section{Hypothesis 2.13.}

1. $\mathcal{D}(A)$ is a linear subspace of the space of $\mathbb{F}^{o}$-progressively measurable processes;

2. A is a linear mapping from $\mathcal{D}(A)$ into the space of $\mathbb{F}^{o}$-progressively measurable processes;

3. for all $\Phi \in \mathcal{D}(A), \omega \in \Omega, \int_{0}^{T}\left|A \Phi_{r}(\omega)\right| d r<+\infty$;

4. for all $\Phi \in \mathcal{D}(A),(s, \eta) \in[0, T] \times \Omega$ and $t \in[s, T]$, we have $\mathbb{E}^{s, \eta}\left[\int_{s}^{t}\left|A(\Phi)_{r}\right| d r\right]<+\infty$ and $\mathbb{E}^{s, \eta}\left[\left|\Phi_{t}\right|\right]<+\infty$.

\section{Definition 2.14.}

1. $\left(\mathbb{P}^{s, \eta}\right)_{(s, \eta) \in[0, T] \times \Omega}$ will be said to solve the martingale problem associated to $(\mathcal{D}(A), A)$ if for every $(s, \eta) \in[0, T] \times \Omega$,

- $\mathbb{P}^{s, \eta}\left(\omega^{s}=\eta^{s}\right)=1$;

- $\Phi-\int_{0}^{\cdot} A(\Phi)_{r} d r$, is on $[s, T]$ a $\left(\mathbb{P}^{s, \eta}, \mathbb{F}^{o}\right)$-martingale for all $\Phi \in \mathcal{D}(A)$.

2. The martingale problem associated to $(\mathcal{D}(A), A)$ will be said to be wellposed if for every $(s, \eta) \in[0, T] \times \Omega$ there exists a unique $\mathbb{P}^{s, \eta}$ verifying both items above.

Inspired from the classical literature (see 13.28 in [18]) we have introduced in [4] the following notion of a weak generator.

Definition 2.15. We say that $(\mathcal{D}(A), A)$ is a weak generator of a pathdependent system of projectors $\left(P_{s}\right)_{s \in[0, T]}$ if for all $\Phi \in \mathcal{D}(A),(s, \eta) \in[0, T] \times \Omega$ and $t \in[s, T]$, we have

$$
P_{s}\left[\Phi_{t}\right](\eta)=\Phi_{s}(\eta)+\int_{s}^{t} P_{s}\left[A(\Phi)_{r}\right](\eta) d r .
$$


The proposition below was the object of Proposition 5.6 in [4].

Proposition 2.16. $(\mathcal{D}(A), A)$ is a weak generator of $\left(P_{s}\right)_{s \in[0, T]}$ if and only if $\left(\mathbb{P}^{s, \eta}\right)_{(s, \eta) \in[0, T] \times \Omega}$ solves the martingale problem associated to $(\mathcal{D}(A), A)$.

In particular, if $\left(\mathbb{P}^{s, \eta}\right)_{(s, \eta) \in[0, T] \times \Omega}$ solves the well-posed martingale problem associated to $(\mathcal{D}(A), A)$ then $\left(P_{s}\right)_{s \in[0, T]}$ is the unique path-dependent system of projectors for which $(\mathcal{D}(A), A)$ is a weak generator.

In the setup of the last statement, one can therefore associate analytically to $(\mathcal{D}(A), A)$ a unique path-dependent system of projectors $\left(P_{s}\right)_{s \in[0, T]}$ through Definition 2.15 .

\section{Path-dependent canonical classes induced by Gaussian measures}

Notation 3.1. Let $(E,\|\cdot\|)$ be a Banach space and $F$ be a linear subspace of $E$ then its closure will be denoted $\bar{F}^{\|\cdot\|}$ or $\bar{F}^{E}$ when there can be no ambiguity concerning the chosen norm.

In this section we will also adopt the conventions of Section 2. Most of the following definitions are taken from [6] Chapter 2.2.

Definition 3.2. Let $\mathbb{P}$ be a Gaussian measure on $(\Omega, \mathcal{F})$, i.e. a probability measure such that for any $n \in \mathbb{N}^{*}$ and $l_{1}, \cdots, l_{n} \in \Omega^{*},\left(l_{1}, \cdots, l_{n}\right)$ has under $\mathbb{P}$ the law of a Gaussian vector. Let $L^{2}(\mathbb{P})$ denote the corresponding space of square integrable random variables and assume that sup $\left\|X_{t}\right\| \in L^{p}(\mathbb{P})$ for all $p \in \mathbb{N}$.

$$
t \in[0, T]
$$

- We define the covariance operator of $\mathbb{P} K: \Omega^{*} \longmapsto \Omega$ by $K l: t \mapsto$ $\mathbb{E}[\omega(t) l(\omega)]$ for all $l \in \Omega^{*}$.

- We denominate covariance function of $\mathbb{P}$ the (symmetric matrix valued) function $c:(s, t) \longmapsto \mathbb{E}\left[X_{s} X_{t}\right]$, and mean function of $\mathbb{P}$ the function $m: t \longmapsto \mathbb{E}\left[X_{t}\right]$. The $(i, j)$-th coordinate of $c$ will be denoted $c_{i, j}$.

- We say that $\mathbb{P}$ is of full support if the smallest closed subset of $\Omega$ of measure 1 is $\Omega$.

- We say that $X$ admits a representation under $\mathbb{P}$ if the following holds. There exists a function $k:[0, T]^{2} \longrightarrow \mathcal{M}_{d}(\mathbb{R})$ such that for all $t \in[0, T]$, $k(t, \cdot) \in L^{2}([0, t])$ and taking value 0 on $\left.] t, T\right]$; and an $\mathbb{F}^{o}$-adapted $\mathbb{R}^{d}$ valued Brownian motion $B:=\left(B^{1}, \cdots, B^{d}\right)$ defined on $(\Omega, \mathcal{F})$ such that

$$
X_{t}=\int_{0}^{t} k(t, r) d B_{r}, \quad \mathbb{P} \text { a.s. }
$$

In this case, $k$ is called the kernel function of $\mathbb{P}$. 
- We call Reproducing Kernel Hilbert Space (RKHS) the Hilbert space of centered elements of $\overline{\operatorname{Span}\left(\left\{X_{r}^{i} \mid i \leq d ; r \in[0, T]\right\}\right)}{ }^{L^{2}(\mathbb{P})}$ which we denote $H(\mathbb{P})$, we also denote for all $t \in[0, T]$ the Hilbert subspace $H^{t}(\mathbb{P})$ of centered elements of $\overline{\operatorname{Span}\left(\left\{X_{r}^{i} \mid i \leq d ; r \in[0, t]\right\}\right)}{ }^{L^{2}(\mathbb{P})}$.

- We call Cameron-Martin space which we denote $\mathcal{H}(\mathbb{P})$, the space of functions $c_{Y}: t \longmapsto \mathbb{E}\left[Y X_{t}\right]$ for $Y \in H(\mathbb{P})$, we equip it with the scalar product defined by $\left(c_{Y}, c_{Z}\right)_{\mathcal{H}(\mathbb{P})}:=\mathbb{E}[Y Z]$ for all $Y, Z \in H(\mathbb{P})$, which makes it a Hilbert space. We also denote for all $t \in[0, T]$ the Hilbert subspace $\mathcal{H}^{t}(\mathbb{P})$ of functions $c_{Y}: t \longmapsto \mathbb{E}\left[Y X_{t}\right]$ for $Y \in H^{t}(\mathbb{P})$.

- We say that $(\mathcal{H}(\mathbb{P}), \Omega, \mathbb{P})$ is an abstract Wiener space if $\mathcal{H}(\mathbb{P})$ is a dense $\left(\right.$ for $\|\cdot\|_{\infty}$ ) subspace of $\Omega$.

Remark 3.3. About Definition 3.2 we mention the following.

- If $\mathbb{P}$ is a Gaussian measure on $(\Omega, \mathcal{F})$ (see Definition 2.2.1 in [6]), then the canonical process $X$ (is under $\mathbb{P}$ ) a Gaussian process;

- $K$ indeed maps $\Omega^{*}$ into $\Omega$ because of Theorem 3.2.3 in [6];

- our definition of $\mathcal{H}(\mathbb{P})$ is not the one of [6] Chapter 2.2, but is equivalent again by Theorem 3.2.3 ibidem, which also ensures that elements of $\mathcal{H}(\mathbb{P})$ belong to $\Omega$;

- $\mathbb{P}$ is of full support if and only if $\mathcal{H}(\mathbb{P})$ is dense in $\Omega$ for \|\|$_{\infty}$ (i.e. $(\mathcal{H}(\mathbb{P}), \Omega, \mathbb{P})$ is an abstract Wiener space), see Theorem 3.6.1 in [6].

We consider a Gaussian probability measure $\mathbb{P}$ on $(\Omega, \mathcal{F})$ verifying the following.

\section{Hypothesis 3.4.}

1. $\sup _{t \in[0, T]}\left\|X_{t}\right\| \in L^{p}(\mathbb{P})$ for every $p \geq 1$;

2. $\mathbb{P}$ is of full support;

3. $X$ admits a representation under $\mathbb{P}$ with respect to some Brownian motion $B$, with a kernel $k$, see (3.1).

4. for all $t \in[0, T]$ and $h \in L^{2}([0, T])$ we have that $\int_{0}^{\cdot} k(\cdot, r) h(r) d r \equiv 0$ on $[0, t]$ implies that $h$ is Lebesgue a.e. equal to zero on $[0, t]$;

5.

$$
M_{o p}:=\max _{i \leq d} \sup _{s \in[0, T] r \in[0, s]} \frac{\max _{j \leq d} \sup \left|\operatorname{sux}_{t \in[0, T]}\right| c_{i, j}(r, t) \mid}{\max _{j^{\prime} \leq d_{r^{\prime} \in[0, s]}} \sup _{i, j^{\prime}}\left(r, r^{\prime}\right) \mid}<+\infty .
$$

Remark 3.5. 
1. Item 5. of Hypothesis 3.4 is verified for example by the following processes.

- Stationary processes, see Corollary 5 in [20];

- the fractional Brownian motion of Hurst index $H \in] 0,1[$, see the proof of Theorem 3.1 in [24].

2. If items 3. and 4. in Hypothesis 3.4 hold, then by Theorem 1.7 in [17], we know that for all $t \in[0, T], H^{t}(\mathbb{P})={\overline{\operatorname{Span}\left(\left\{B_{r}^{i} \mid i \leq d ; r \in[0, t]\right\}\right)}}^{L^{2}(\mathbb{P})}$.

\section{Notation 3.6.}

- For all $s \in[0, T]$, we denote by $\Omega_{s}$ the Banach subspace of $\Omega$ constituted of paths $\omega$ constant after time $s$, i.e. such that $\omega=\omega^{s}$ and we denote by $\pi_{s}$ the continuous mapping $\begin{aligned} \Omega & \longrightarrow \Omega_{s} \\ \omega & \longmapsto \omega^{s} .\end{aligned}$

- By a slight abuse of notation, we denote by $\Omega_{s}^{\perp}$ the Banach subspace $\Omega$ constituted of paths $\omega$ taking value 0 on $[0, s]$, and by $\pi_{s}^{\perp}$ the continuous mapping $\Omega \longrightarrow \Omega_{s}^{\perp}$

- $K: \Omega^{*} \longrightarrow \Omega$ denotes the covariance operator of $\mathbb{P}$, see Definition 3.2.

- Let $k$ be the function appearing in (3.1), then for all $i \leq d, k_{i}:[0, T] \times$ $[0, T] \rightarrow \mathbb{R}^{d}$ will denote its $i$-th column, and for all $i, j \leq d, k_{i, j}:[0, T] \times$ $[0, T] \rightarrow \mathbb{R}$ will denote its $(i, j)$-th coefficient.

In the proposition below for every $s \in[0, T], \eta \in \Omega_{s}$ we introduce a Gaussian probability measure $P^{s, \eta}$ which represents the conditional law of (3.1) given $\omega^{s}=\eta^{s}$.

\section{Proposition 3.7.}

1. $K \Omega^{*}$ is dense in $\Omega$.

2. For every $s \in[0, T]$, there exists a set of Gaussian probability measures $\left(\mathbb{P}^{s, \eta}\right)_{\eta \in \Omega_{s}}$ (with related expectations $\left(\mathbb{E}^{s, \eta}\right)_{\eta \in \Omega_{s}}$ ) and a continuous operator $m_{s}: \Omega_{s} \longrightarrow \Omega$ such that the following holds.

(a) For all $\eta \in \Omega_{s}, \mathbb{P}^{s, \eta}\left(\omega^{s}=\eta\right)=1$;

(b) $\eta \longmapsto \mathbb{P}^{s, \eta}$ is continuous;

(c) for every $t \geq s$ and $F \in \mathcal{F}$,

$$
\mathbb{P}\left(F \mid \mathcal{F}_{s}^{o}\right)(\eta)=\mathbb{P}^{s, \eta}(F) \text { for } \mathbb{P} \text { almost all } \eta
$$

(d) for all $t \in[0, T], \mathbb{E}^{s, \eta}\left[X_{t}\right]=m_{s}[\eta](t)$;

(e) for all $s, m_{s}$ has an operator norm inferior to $M_{o p}$; 
(f) $\pi_{s} K \pi_{s}^{*} \Omega_{s}^{*}$ is dense in $\Omega_{s}$ and that on $K \pi_{s}^{*} \Omega_{s}^{*}, m_{s} \circ \pi_{s}$ coincides with the identity.

Proof. The statement 1 . follows from Theorem 2.1 in [20] and the fact that by Hypothesis 3.4 item 2., the support of $\mathbb{P}$ is $\Omega$.

The statement 2. follows from Theorem 2.1 and Theorem 2 and the statement and proof of Lemma 2.2 in [20] applied for fixed $s$ to the continuous linear operator $\pi_{s}$ between the Banach spaces $\Omega$ and $\Omega_{s}$.

Definition 3.8. 1 . For every $s \in[0, T]$, we extend $\eta \mapsto m_{s}[\eta]$ and $\eta \mapsto \mathbb{P}^{s, \eta}$ from $\Omega_{s}$ to $\Omega$ by setting for all $\eta \in \Omega, m_{s}[\eta]:=m_{s}\left[\eta^{s}\right]$ and $\mathbb{P}^{s, \eta}:=\mathbb{P}^{s, \eta^{s}}$.

2. $(s, \eta) \mapsto m_{s}[\eta]$ will be called the mean random field.

By continuity of $\pi_{s}$, we remark that for all $s, \eta \mapsto m_{s}[\eta], \eta \longmapsto \mathbb{P}^{s, \eta}$ remain continuous.

Remark 3.9. The family of linear operators $\left(m_{s}\right)_{s \in[0, T]}$ constituting the mean random field is crucial in this paper.

1. First, that consitutes a very useful analytical tool, since it has permit to [20] to prove that the regular conditional expectation of $\mathbb{P}$ is continuous, see Proposition 3.7 item 2.b.

2. Second, it is also central at the probabilistic level of this paper. In particular $m_{s}[\eta]$ is the mean function of $\mathbb{P}^{s, \eta}$, the mean random field will also allow us to construct driving martingales for our BSDEs, as we will see in item 3. of Proposition 4.20.

The proof of Propositions 3.10 and 3.11 below is postponed to the Appendix.

Proposition 3.10. $\left(\mathbb{P}^{s, \eta}\right)_{(s, \eta) \in[0, T] \times \Omega}$ is a path-dependent canonical class.

Proposition 3.11. For all $\eta \in \Omega, s \longmapsto m_{s}[\eta]$ is right-continuous in $\left(\Omega,\|\cdot\|_{\infty}\right)$, in particular, for all $t \in[0, T] s \longmapsto m_{s}[\eta](t)$ is right-continuous.

Corollary 3.12. $m: \begin{aligned}(s, \eta) & \longmapsto m_{s}[\eta] \\ {[0, T] \times \Omega } & \longrightarrow \Omega\end{aligned}$ is $\left(\right.$ Pro $\left.^{o}, \mathcal{F}\right)$-measurable.

Moreover, for all $t \in[0, T], \quad \begin{aligned}(s, \eta) & \longmapsto m_{s}[\eta](t)\end{aligned}$ is $\mathbb{F}^{o}$-progressively measurable.

Proof. Let $T_{0} \in[0, T] . m:\left[0, T_{0}\right] \times \Omega_{T_{0}} \mapsto \Omega$ is right-continuous in $s \in\left[0, T_{0}\right]$ at fixed $\eta \in \Omega_{T_{0}}$ by Proposition 3.11 and continuous in $\eta$ at fixed $s$ hence jointly measurable, see Theorem 15 in [9] Chapter IV.

For all $t \leq T_{0}$ and $\omega \in \Omega$, we have $m_{t}(\omega)=m_{t} \circ \pi_{t}(\omega)=m_{t} \circ \pi_{T_{0}}(\omega)$, taking into account Definition 3.8. 
So $m:\left[0, T_{0}\right] \times \Omega \mapsto \Omega$ can be expressed composing $m:\left[0, T_{0}\right] \times \Omega_{T_{0}} \mapsto \Omega$ with

$$
\begin{aligned}
(t, \omega) & \mapsto\left(t, \pi_{T_{0}}(\omega)\right) \\
{\left[0, T_{0}\right] \times \Omega } & \rightarrow\left[0, T_{0}\right] \times \Omega_{T_{0}}
\end{aligned},
$$

which is clearly $\left(\mathcal{B}\left(\left[0, T_{0}\right]\right) \otimes \mathcal{F}_{T_{0}}^{o}, \mathcal{B}\left(\left[0, T_{0}\right]\right) \otimes \mathcal{B}\left(\Omega_{T_{0}}\right)\right)$-measurable. By composition, $m:\left[0, T_{0}\right] \times \Omega \mapsto \Omega$ is $\mathcal{B}\left(\left[0, T_{0}\right]\right) \otimes \mathcal{F}_{T_{0}}^{o}$-measurable. Since this holds for all $T_{0}$, the first statement is shown.

The second part of the statement follows composing $m_{s}(\eta)$ and $X_{t}$ which is continuous hence measurable for all $t$.

Remark 3.13. For every $(s, \eta), X-m_{s}[\eta]$ is under $\mathbb{P}^{s, \eta}$ a mean-zero continuous Gaussian process whose covariance function does not depend on $\eta$, see Theorem 2 in [20].

In particular, if $s$ is fixed and if we consider two paths $\eta, \eta^{\prime}$ in $\Omega$, then $\mathbb{P}^{s, \eta^{\prime}}$ is the translation of $\mathbb{P}^{s, \eta}$ by the vector $m_{s}[\eta]-m_{s}\left[\eta^{\prime}\right]=m_{s}\left[\eta-\eta^{\prime}\right]$.

Notation 3.14. For every $(s, \eta)$, we denote by $c^{s}$ the covariance function of $X-m_{s}[\eta]$ under $\mathbb{P}^{s, \eta}$. We denote by $\mathbb{P}^{s}$ the law of that process, which does not depend on $\eta$. The expectation under $\mathbb{P}^{s}$ will be denoted by $\mathbb{E}^{s}$.

Proposition 3.15. For every $(s, \eta) \in[0, T] \times \Omega$ and $p \in \mathbb{N}^{*}, \sup _{r \in[0, T]}\left\|X_{r}\right\| \in$ $\mathcal{L}^{p}\left(\mathbb{P}^{s, \eta}\right)$.

Proof. We fix $s \in[0, T]$ and $p \in \mathbb{N}^{*}$. We start by noticing that for every $\eta \in \Omega$,

$$
\mathbb{E}^{s, \eta}\left[\|\omega\|_{\infty}^{p}\right]=\mathbb{E}^{s}\left[\left\|\omega+m_{s}[\eta]\right\|_{\infty}^{p}\right] .
$$

Then by triangle inequality for $\|\cdot\|_{\infty}$ and convexity of $x \mapsto x^{p}$, we can write

$$
\left\|\omega+m_{s}[\eta]\right\|_{\infty}^{p} \leq 2^{p-1}\|\omega\|_{\infty}^{p}+2^{p-1}\left\|m_{s}[\eta]\right\|_{\infty}^{p} .
$$

Since $\|\omega\|_{\infty}^{p}=\left\|-m_{s}[\eta]+\left(\omega+m_{s}[\eta]\right)\right\|_{\infty}^{p} \leq 2^{p-1}\left\|m_{s}[\eta]\right\|_{\infty}^{p}+2^{p-1}\left\|\omega+m_{s}[\eta]\right\|_{\infty}^{p}$ then

$$
\frac{1}{2^{p-1}}\|\omega\|_{\infty}^{p}-\left\|m_{s}[\eta]\right\|_{\infty}^{p} \leq\left\|\omega+m_{s}[\eta]\right\|_{\infty}^{p} .
$$

Taking the expectation $\mathbb{E}^{s}$ in (3.5), (3.6) and taking (3.4) into account yields

$$
\frac{1}{2^{p-1}} \mathbb{E}^{s}\left[\|\omega\|_{\infty}^{p}\right]-\left\|m_{s}[\eta]\right\|_{\infty}^{p} \leq \mathbb{E}^{s, \eta}\left[\|\omega\|_{\infty}^{p}\right] \leq 2^{p-1} \mathbb{E}^{s}\left[\|\omega\|_{\infty}^{p}\right]+2^{p-1}\left\|m_{s}[\eta]\right\|_{\infty}^{p} .
$$

So either $\mathbb{E}^{s}\left[\|\omega\|_{\infty}^{p}\right]$ is finite and therefore $\mathbb{E}^{s, \eta}\left[\|\omega\|_{\infty}^{p}\right]$ is finite for all $\eta$, or $\mathbb{E}^{s}\left[\|\omega\|_{\infty}^{p}\right]$ is infinite and therefore $\mathbb{E}^{s, \eta}\left[\|\omega\|_{\infty}^{p}\right]$ is infinite for all $\eta$. We now show that the second option is not possible in order to conclude. Indeed, by Remark 2.5 we have

$$
\begin{aligned}
\mathbb{E}\left[\|\omega\|_{\infty}^{p}\right] & =\mathbb{E}\left[\mathbb{E}\left[\|\omega\|_{\infty}^{p} \mid \mathcal{F}_{s}^{o}\right](\eta)\right] \\
& =\mathbb{E}\left[\mathbb{E}^{s, \eta}\left[\|\omega\|_{\infty}^{p}\right]\right],
\end{aligned}
$$

where we recall in particular that $\mathbb{E}\left[\|\omega\|_{\infty}^{p}\right]<+\infty$ by Hypothesis 3.4 , so $\mathbb{E}^{s, \eta}\left[\|\omega\|_{\infty}^{p}\right.$ is finite for $\mathbb{P}$ almost all $\eta$ hence is not infinite for all $\eta$. 
The proposition below is proved in the Appendix.

Proposition 3.16. For every $(s, \eta) \in[0, T] \times \Omega$, the topological support of $\mathbb{P}^{s, \eta}$ is equal to $\eta^{s}+\Omega_{s}^{\perp}$ i.e. the set of paths coinciding with $\eta$ on $[0, s]$.

We recall that the covariance functions $c^{s}$ have been defined at Notation 3.14 .

Lemma 3.17. For every $s, t, u \in[0, T]$,

$$
c^{s}(t, u)=\left\{\begin{array}{l}
\int_{s}^{t \wedge u} k(t, r) k(u, r) d r \quad \text { if } s \leq t, u \\
0 \quad \text { otherwise. }
\end{array}\right.
$$

Proof. We fix $t, u$. For every $\eta \in \Omega$, we have

$$
c^{s}(t, u)=\mathbb{E}^{s, \eta}\left[X_{t} \otimes X_{u}\right]-\mathbb{E}^{s, \eta}\left[X_{t}\right] \otimes \mathbb{E}^{s, \eta}\left[X_{u}\right] .
$$

Clearly if $t$ (resp. $u$ ) is inferior to $s$ then $X_{t}$ (resp. $X_{u}$ ) is for all $\eta \mathbb{P}^{s, \eta}$ a.s. deterministic, see Proposition 3.7 2. (a). This implies $c^{s}(t, u)=0$. Assume now that $s \leq t, u$. By Proposition 3.7 2. (c) and (3.10) we have $\mathbb{P}$ a.s. that,

$$
\begin{aligned}
c^{s}(t, u)= & \mathbb{E}\left[X_{t} \otimes X_{u} \mid \mathcal{F}_{s}^{o}\right]-\mathbb{E}\left[X_{t} \mid \mathcal{F}_{s}^{o}\right] \otimes \mathbb{E}\left[X_{u} \mid \mathcal{F}_{s}^{o}\right] \\
= & \mathbb{E}\left[\left(\int_{0}^{t} k(t, r) d B_{r}\right) \otimes\left(\int_{0}^{u} k(u, r) d B_{r}\right) \mid \mathcal{F}_{s}^{o}\right] \\
& -\mathbb{E}\left[\int_{0}^{t} k(t, r) d B_{r} \mid \mathcal{F}_{s}^{o}\right] \otimes \mathbb{E}\left[\int_{0}^{t} k(t, r) d B_{r} \mid \mathcal{F}_{s}^{o}\right] \\
= & \left(\int_{0}^{s} k(t, r) d B_{r}\right) \otimes\left(\int_{0}^{s} k(t, r) d B_{r}\right)+\int_{s}^{t \wedge u} k(t, r) k(u, r) d r \\
& -\left(\int_{0}^{s} k(t, r) d B_{r}\right) \otimes\left(\int_{0}^{s} k(t, r) d B_{r}\right) \\
= & \int_{s}^{t \wedge u} k(t, r) k(u, r) d r,
\end{aligned}
$$

and the proof is complete.

The proof of the proposition below is also located in the Appendix.

Proposition 3.18. $\left(\mathbb{P}^{s, \eta}\right)_{(s, \eta) \in[0, T] \times \Omega}$ is progressive, see Definition 2.4.

Notation 3.19. For any $(s, \eta) \in[0, T] \times \Omega$ we will consider the stochastic basis $\left(\Omega, \mathcal{F}^{s, \eta}, \mathbb{F}^{s, \eta}:=\left(\mathcal{F}_{t}^{s, \eta}\right)_{t \in[0, T]}, \mathbb{P}^{s, \eta}\right)$ where $\mathcal{F}^{s, \eta}$ (resp. $\mathcal{F}_{t}^{s, \eta}$ for all $t$ ) is $\mathcal{F}$ (resp. $\mathcal{F}_{t}$ ) augmented with the $\mathbb{P}^{s, \eta}$ negligible sets. $\mathbb{P}^{s, \eta}$ is extended to $\mathcal{F}^{s, \eta}$.

We remark that, for any $(s, \eta) \in[0, T] \times \Omega,\left(\Omega, \mathcal{F}^{s, \eta}, \mathbb{F}^{s, \eta}, \mathbb{P}^{s, \eta}\right)$ is a stochastic basis fulfilling the usual conditions, see 1.4 in [18] Chapter I.

Proposition 3.20. Let $X^{s, \eta}$ denote the process $X-m_{s}[\eta]$. Then there exists an $\mathbb{R}^{d}$-valued $\left(\mathbb{P}^{s, \eta}, \mathbb{F}^{s, \eta}\right)$-Brownian motion $B^{s, \eta}$ starting in $s$ such that for all $t \geq s, X_{t}^{s, \eta}=\int_{s}^{t} k(t, r) d B_{r}^{s, \eta} \mathbb{P}^{s, \eta}$ a.s. Moreover, for all $t \geq s$, then $\mathcal{F}_{t}^{s, \eta}$, coincides with $\mathcal{F}_{t}^{o}$ augmented with $\mathbb{P}^{s, \eta}$-null sets.

Corollary 3.21. $\left(\mathbb{P}^{s, \eta}\right)_{(s, \eta) \in[0, T] \times \Omega}$ verifies Hypothesis 2.6.

Proof. By Proposition $3.10\left(\mathbb{P}^{s, \eta}\right)$ is a path-dependent canonical class, in particular (2.1) holds. Taking into account Notation 3.19 the result follows by Proposition 3.20 . 
We now conclude this section by extending previous results to the case with drift. In Proposition 3.22 below, $\mathbb{Q}^{s, \eta}$ will model the conditional law of $\beta+$ $\int_{0}^{\cdot} k(\cdot, r) d B_{r}$ given $\omega^{s}=\eta^{s}$ where $\beta$ is a given path in $\Omega$.

Proposition 3.22. Let $\beta \in \Omega$ and define for all $(s, \eta)$, and $F \in \mathcal{F}: \mathbb{Q}^{s, \eta}(F):=$ $\mathbb{P}^{s, \eta-\beta^{s}}(F-\beta)$.

Then, the following holds.

1. $\left(\mathbb{Q}^{s, \eta}\right)_{(s, \eta) \in[0, T] \times \Omega}$ is a progressive path-dependent canonical class satisfying Hypothesis 2.6.

2. For all $(s, \eta), \mathbb{Q}^{s, \eta}$ is a Gaussian measure with mean function $\beta+m_{s}[\eta-\beta]$ and covariance function $c^{s}$, see Definition 3.2.

3. For all $(s, \eta)$, under $\mathbb{Q}^{s, \eta}$, there exists a Brownian motion $\tilde{B}^{s, \eta}$ such that on $[s, T], X$ is indistinguishable from $m_{s}[\eta-\beta]+\beta+\int_{s}^{\cdot} k(\cdot, r) d \tilde{B}_{r}^{s, \eta}$.

Proof. We start with the first statement. The progressivity property follows by the one of $\left(\mathbb{P}^{s, \eta}\right)_{s, \eta}$. Since $\left(\mathbb{P}^{s, \eta}\right)_{s, \eta}$ is a path-dependent canonical class items 1. and 2. of Definition 2.4 are clearly verified, so we only have to show that (2.2) holds. We fix $(s, \eta), t \geq s, F \in \mathcal{F}$ and we show that

$$
\mathbb{Q}^{s, \eta}\left(F \mid \mathcal{F}_{t}\right)=\mathbb{Q}^{t, \omega}(F), \quad \mathbb{Q}^{s, \eta}, \text { a.s. }
$$

Let $G \in \mathcal{F}_{t}$. We have

$$
\begin{aligned}
\mathbb{E}^{\mathbb{Q}^{s, \eta}}\left[\mathbb{1}_{F}(\omega) \mathbb{1}_{G}(\omega)\right] & =\mathbb{E}^{s, \eta-\beta^{s}}\left[\mathbb{1}_{F-\beta}(\omega) \mathbb{1}_{G-\beta}(\omega)\right] \\
& =\mathbb{E}^{s, \eta-\beta^{s}}\left[\mathbb{E}^{s, \eta-\beta^{s}}\left[\mathbb{1}_{F-\beta} \mid \mathcal{F}_{t}\right](\omega) \mathbb{1}_{G-\beta}(\omega)\right] \\
& =\mathbb{E}^{s, \eta-\beta^{s}}\left[\mathbb{P}^{t, \omega}(F-\beta) \mathbb{1}_{G-\beta}(\omega)\right] \\
& =\mathbb{E}^{s, \eta-\beta^{s}}\left[\mathbb{Q}^{t, \omega+\beta^{t}}(F) \mathbb{1}_{G-\beta}(\omega)\right] \\
& =\mathbb{E}^{\mathbb{Q}^{s, \eta}}\left[\mathbb{Q}^{t, \omega}(F) \mathbb{1}_{G}(\omega)\right],
\end{aligned}
$$

where the second equality holds because $G-\beta \in \mathcal{F}_{t}$; the third equality because $\left(\mathbb{P}^{s, \eta}\right)_{(s, \eta) \in[0, T] \times \Omega}$ verifies Hypothesis 2.6 (see Corollary 3.21) and the last two equalities by definition of the $\mathbb{Q}^{s, \eta}$.

By definition of conditional expectation, the fact that (3.13) holds for all $G \in \mathcal{F}_{t}$ implies (3.12).

Concerning the second statement, we fix $(s, \eta)$. $\mathbb{Q}^{s, \eta}$ is the translation of $\mathbb{P}^{s, \eta-\beta^{s}}$ in the direction $\beta$, so it is a Gaussian measure with same covariance function $c^{s}$ and with mean function the mean function (see Definition 3.2) of $\mathbb{P}^{s, \eta-\beta^{s}}$, translated of $\beta$, meaning $m_{s}[\eta-\beta]+\beta$.

Finally the third statement is a consequence of Proposition 3.20 and of item 2 . 


\section{BSDEs with Gaussian forward process and de- coupled mild solutions of path-dependent PDEs}

\subsection{General considerations.}

This section is the main part of the paper. Its aim is to introduce formally equation $P D P D E(f, \xi)$ introduced in the introduction (see (1.6)), its coefficients, the operators that it involves, and to prove existence and uniqueness of what we call a decoupled mild solution. We will make use of the probabilistic framework and results obtained in the previous section.

We are now given a Gaussian measure $\mathbb{P}$ satisfying Hypothesis 3.4 and the corresponding path-dependent canonical class $\left(\mathbb{P}^{s, \eta}\right)_{(s, \eta) \in[0, T] \times \Omega}$, see Proposition 3.10 .

We fix a function $b:[0, T] \times[0, T] \rightarrow \mathbb{R}^{d}$ and we assume for the remainder of the paper that $b, k$ verify the following.

\section{Hypothesis 4.1.}

- $b, k$ are bounded Borel;

- for all $s \in[0, T], b(s, \cdot)$ and $k(s, \cdot)$ are equal to 0 on $[0, s[$ and continuous on $[s, T]$ where they admit a bounded right-derivative;

- $t \mapsto \int_{0}^{t} b(t, r) d r$ is continuous.

Definition 4.2. We set $\beta: t \mapsto \int_{0}^{t} b(t, r) d r$ and define $\left(\mathbb{Q}^{s, \eta}\right)_{(s, \eta) \in[0, T] \times \Omega}$ as in Proposition 3.22 with this specific choice of $\beta$.

Notation 4.3. In this section, the continuous operator $m_{s}[\cdot-\beta]$ will be denoted $m_{s}$ and $\mathbb{E}^{s, \eta}$ will denote the expectation with respect to $\mathbb{Q}^{s, \eta}$ and not $\mathbb{P}^{s, \eta}$ any more.

We recall that by Proposition 3.22, we have the following.

\section{Remark 4.4.}

- $\left(\mathbb{Q}^{s, \eta}\right)_{(s, \eta) \in[0, T] \times \Omega}$ defines a progressive path-dependent canonical class verifying Hypothesis 2.6;

- for every $(s, \eta), \mathbb{Q}^{s, \eta}$ is the Gaussian measure on $(\Omega, \mathcal{F})$ of covariance function $c^{s}$ and mean function $m_{s}[\eta]+\beta$, see Definition 3.2;

- for every $(s, \eta)$, there exists a $\mathbb{Q}^{s, \eta}$-Brownian motion $B^{s, \eta}$ such that under $\mathbb{Q}^{s, \eta}$ we have

$$
X=m_{s}[\eta]+\int_{0}^{\cdot} b(\cdot, r) d r+\int_{s} k(\cdot, r) d B_{r}^{s, \eta},
$$

on $[s, T]$. 


\subsection{Differential operators involved in the path-dependent PDE}

Notation 4.5. From now on, $\left(P_{s}\right)_{s \in[0, T]}$ denotes the path-dependent system of projectors associated (in the sense of Definition 2.10) to $\left(\mathbb{Q}^{s, \eta}\right)_{(s, \eta) \in[0, T] \times \Omega}$.

Our aim now is to provide a weak generator $(\mathcal{D}(A), A)$ of $\left(P_{s}\right)_{s \in[0, T]}$, see Definition 2.15.

The following definitions are adapted from [26] Section 3.1.

Definition 4.6. We denote by $\bar{\Omega}:=\mathbb{D}([0, T], \mathbb{R})$ the Skorohod space of cadlag functions from $[0, T]$ to $\mathbb{R}$. $\overline{\mathcal{F}}$ (resp. $\overline{\mathbb{F}}^{o}$ ) will denote the corresponding Borel $\sigma$-field (resp. initial filtration, see Notation 2.2).

Let $t \in[0, T] . \bar{\Omega}_{t}$ will denote the set of elements of $\bar{\Omega}$ equal to 0 on $[0, t[$ and continuous on $[t, T]$. For $\omega, \eta \in \bar{\Omega}$. $\eta \otimes_{t} \omega$ will denote $\eta \mathbb{1}_{[0, t[}+\omega \mathbb{1}_{[t, T[}$.

$\bar{\Lambda}$ will denote the set of $(t, \omega) \in[0, T] \times \bar{\Omega}$ such that $\omega$ is continuous after time t. We equip $\bar{\Lambda}$ with the distance defined by $d((s, \eta),(t, \omega))=|t-s|+\|\omega-\eta\|_{\infty}$. $\mathcal{C}^{0}(\bar{\Lambda})$ will denote the set of real-valued functions on $\bar{\Lambda}$, continuous with respect to $d$.

We fix $\Phi \in \mathcal{C}^{0}(\bar{\Lambda})$. For $(t, \omega) \in \bar{\Lambda}, D \Phi_{t}(\omega)$ will denote $\lim _{\epsilon \rightarrow 0+} \frac{1}{\epsilon}\left(\Phi_{t+\epsilon}(\omega)-\Phi_{t}(\omega)\right)$ if this limit exists.

Let $\eta \in \bar{\Omega}_{t}, \nabla_{\eta} \Phi_{t}(\omega)$ will denote $\lim _{\epsilon \rightarrow 0} \frac{1}{\epsilon}\left(\Phi_{t}(\omega+\epsilon \eta)-\Phi_{t}(\omega)\right)$ if this limit exists. We define $\nabla_{\eta}^{2} \Phi_{t}(\omega)$ similarly for $\eta \in \bar{\Omega}_{t}$.

We say that $\Phi$ has polynomial growth if there exists $C>0, p \geq 1$ such that $\left|\Phi_{t}(\omega)\right| \leq C\left(1+\|\omega\|_{\infty}^{p}\right)$ for all $(t, \omega) \in \bar{\Lambda}$.

Concerning gradient processes, we will say that $\nabla \Phi$ has polynomial growth if there exists $C>0, p \geq 1$ such that $\left|\nabla_{\eta} \Phi_{t}(\omega)\right| \leq C\left(1+\|\omega\|_{\infty}^{p}\right)$ for all $(t, \omega) \in \bar{\Lambda}$ and $\eta \in \bar{\Omega}_{t}$.

We say that $\nabla \Phi$ is continuous if for all $\eta \in \bar{\Omega}_{t},(t, \omega) \mapsto \nabla_{\eta} \Phi_{t}(\omega) \in \mathcal{C}^{0}(\bar{\Lambda})$.

Finally we define $\mathcal{C}_{+}^{1,2}(\bar{\Lambda})$ the set of elements $\Phi \in \mathcal{C}^{0}(\bar{\Lambda})$ verifying the following hypothesis.

- $D \Phi, \nabla \Phi, \nabla^{2} \Phi$ exist and are continuous;

- $\Phi, D \Phi, \nabla \Phi, \nabla^{2} \Phi$ have polynomial growth;

- there exists $p \geq 1$ and a bounded modulus of continuity $\rho$ such that for all $(t, \omega),\left(t, \omega^{\prime}\right) \in \bar{\Lambda}$ and $\eta \in \bar{\Omega}_{t}$,

$$
\left|\nabla_{\eta}^{2} \Phi_{t}(\omega)-\nabla_{\eta}^{2} \Phi_{t}\left(\omega^{\prime}\right)\right| \leq\left(1+\|\omega\|_{\infty}^{p}+\left\|\omega^{\prime}\right\|_{\infty}^{p}\right) \rho\left(\left\|\omega-\omega^{\prime}\right\|_{\infty}\right) .
$$

In the sequel, given $\tilde{\Phi} \in \mathcal{C}^{0}(\bar{\Lambda})$, we will denote

$$
\Phi:(t, \omega) \longmapsto \tilde{\Phi}_{t}\left(m_{t}[\omega]\right) .
$$

Lemma 4.7. Let $\tilde{\Phi} \in \mathcal{C}^{0}(\bar{\Lambda})$. Then the following holds. 
1. $\Phi$ is $\mathbb{F}^{o}$-progressively measurable.

2. If moreover $\tilde{\Phi} \in \mathcal{C}_{+}^{1,2}(\bar{\Lambda}),(t, \omega) \mapsto D \tilde{\Phi}_{t}\left(m_{t}[\omega]\right),(t, \omega) \mapsto \nabla_{b(\cdot, t)} \tilde{\Phi}_{t}\left(m_{t}[\omega]\right)$; $(t, \omega) \mapsto \nabla_{k_{i}(\cdot, t)}^{2} \tilde{\Phi}_{t}\left(m_{t}[\omega]\right), i \leq d$ are also $\mathbb{F}^{o}$-progressively measurable.

Proof. 1. We fix $\tilde{\Phi} \in \mathcal{C}^{0}(\bar{\Lambda})$. By Corollary 3.12, $(t, \omega) \mapsto m_{t}[\omega]$ is $\mathbb{F}^{o}$

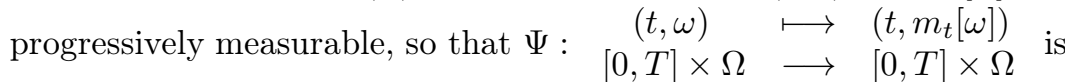

$\left(\mathcal{P r o}{ }^{o}, \mathcal{B}([0, T]) \otimes \mathcal{F}\right)$-measurable. We remark that $[0, T] \times \Omega$ is a subset of $\bar{\Lambda}$. The restriction of $\tilde{\Phi}$ to $[0, T] \times \Omega$ of $\bar{\Lambda}$ is continuous for the usual topology hence $(\mathcal{B}([0, T]) \otimes \mathcal{F}, \mathcal{B}(\mathbb{R}))$-measurable. By composition, $\Phi=\tilde{\Phi} \circ \Psi$ is $\left(\mathcal{P r o}^{\circ}, \mathcal{B}(\mathbb{R})\right)$-measurable.

2. We now discuss the statement 2. Since $D \tilde{\Phi} \in \mathcal{C}^{0}(\bar{\Lambda})$, by the statement 1. of the lemma that $(t, \omega) \mapsto D \tilde{\Phi}_{t}\left(m_{t}[\omega]\right)$ is progressively measurable. We will now show that the same holds for the first order space derivative $(t, \omega) \mapsto \nabla_{b(\cdot, t)} \tilde{\Phi}_{t}\left(m_{t}[\omega]\right)$.

Since $\tilde{\Phi}$ is in $\mathcal{C}_{+}^{1,2}(\bar{\Lambda})$, then by definition, for all $\eta,(t, \omega) \mapsto \nabla_{\eta} \tilde{\Phi}_{t}(\omega)$ is continuous. On the other hand, it is clear that for all $(t, \omega), \eta \mapsto \nabla_{\eta} \tilde{\Phi}_{t}(\omega)$ is measurable as the limit of measurable mappings. So $(\eta, t, \omega) \mapsto \nabla_{\eta} \tilde{\Phi}_{t}(\omega)$ is jointly measurable, see Lemma 4.51 in [1]. Since $b$ is Borel, then $t \mapsto b^{t}$ is Borel from $([0, T], \mathcal{B}([0, T]))$ into $(\bar{\Omega}, \overline{\mathcal{F}})$. By composition, we get that $(t, \omega) \mapsto \nabla_{b(\cdot, t)} \tilde{\Phi}_{t}(\omega)$ is measurable. We can now conclude as for the first statement by composing with $(t, \omega) \mapsto\left(t, m_{t}(\omega)\right)$.

Finally, similar arguments allow to show the progressive measurability of the second order space derivatives $(t, \omega) \mapsto \nabla_{k_{i}(\cdot, t)}^{2} \tilde{\Phi}_{t}\left(m_{t}[\omega]\right), i \leq d$.

Definition 4.8. We denote $\mathcal{D}(\tilde{A})$ to be the set of mappings $\tilde{\Phi} \in \mathcal{C}_{+}^{1,2}(\bar{\Lambda})$ such that $(t, \omega) \mapsto D \tilde{\Phi}_{t}\left(m_{t}[\omega]\right),(t, \omega) \mapsto \nabla_{b(\cdot, t)} \tilde{\Phi}_{t}\left(m_{t}[\omega]\right) ;(t, \omega) \mapsto \nabla_{k_{i}(\cdot, t)} \tilde{\Phi}_{t}\left(m_{t}[\omega]\right), i \leq$ $d,(t, \omega) \mapsto \nabla_{k_{i}(\cdot, t)}^{2} \tilde{\Phi}_{t}\left(m_{t}[\omega]\right), i \leq d$ have polynomial growth. On that space we define the linear operator $\tilde{A}$ by setting, for all $\tilde{\Phi} \in \mathcal{D}(\tilde{A})$ and $t \in[0, T]$,

$$
\tilde{A} \tilde{\Phi}_{t}:=D \tilde{\Phi}_{t}+\nabla_{b(\cdot, t)} \tilde{\Phi}_{t}+\frac{1}{2} \sum_{i \leq d} \nabla_{k_{i}(\cdot, t)}^{2} \tilde{\Phi}_{t} .
$$

We then denote $\mathcal{D}(A)$ to be the set of processes $\Phi:(t, \omega) \longmapsto \tilde{\Phi}_{t}\left(m_{t}[\omega]\right)$ where $\tilde{\Phi} \in \mathcal{D}(\tilde{A})$, and $A$ to be the linear operator defined for all $\Phi:(t, \omega) \longmapsto$ $\tilde{\Phi}_{t}\left(m_{t}[\omega]\right) \in \mathcal{D}(A)$ by

$$
A \Phi_{t}(\omega):=\tilde{A} \tilde{\Phi}_{t}\left(m_{t}(\omega)\right), \forall(t, \omega) .
$$

Remark 4.9. $\mathcal{C}_{+}^{1,2}(\bar{\Lambda}), \mathcal{D}(\tilde{A})$ and $\mathcal{D}(A)$ are linear algebras.

Proposition 4.10. $(\mathcal{D}(A), A)$ introduced in previous Definition 4.8 fulfills Hypothesis 2.13. 
Proof. Items 1. and 2. of Hypothesis 2.13 are fulfilled thanks to Lemma 4.7; items 3. and 4. follow from polynomial growth of $\Phi, A \Phi$ for all $\Phi \in \mathcal{D}(A)$ and the fact that for all $s, \eta, p \geq 1$, $\sup _{t \in[0, T]}\left|X_{t}\right| \in L^{p}\left(\mathbb{Q}^{s, \eta}\right)$, see Proposition 3.15 and the fact that $\mathbb{Q}^{s, \eta}$ is a translation of $\mathbb{P}^{s}$.

The next step consists in proving that $\left(\mathbb{Q}^{s, \eta}\right)_{(s, \eta) \in[0, T] \times \Omega}$ solves the martingale problem associated to $(\mathcal{D}(A), A)$, see Definition 2.14. Indeed by Remark 4.4 , for all $(s, \eta)$, under $\mathbb{Q}^{s, \eta}$, the process $\tilde{X}^{s, \eta}:=X-m_{s}[\eta]$ solves the Volterra SDE

$$
\tilde{X}_{t}^{s, \eta}=\int_{s}^{t} b(t, r) d r+\int_{s}^{t} k(t, r) d B_{r}^{s, \eta}, \quad t \in[s, T] .
$$

In this framework Theorem 3.9 in [26] implies the following chain rule formula.

Theorem 4.11. For every $(s, \eta) \in[0, T] \times \Omega$ and $\tilde{\Phi} \in \mathcal{C}_{+}^{1,2}(\bar{\Lambda})$ we have the following. For all $t \geq s$

$$
\begin{aligned}
\tilde{\Phi}_{t}\left(m_{t}[\omega]-m_{s}[\eta]\right)= & \tilde{\Phi}_{s}(0)+\int_{s}^{t} D \tilde{\Phi}_{r}\left(m_{r}[\omega]-m_{s}[\eta]\right) d r \\
& +\int_{s}^{t} \nabla_{b(\cdot, r)} \tilde{\Phi}_{r}\left(m_{r}[\omega]-m_{s}[\eta]\right) d r \\
& +\frac{1}{2} \sum_{i \leq d} \int_{s}^{t} \nabla_{k_{i}(\cdot, r)}^{2} \tilde{\Phi}_{r}\left(m_{r}[\omega]-m_{s}[\eta]\right) d r \\
& +\sum_{i \leq d} \int_{s}^{t} \nabla_{k_{i}(\cdot, r)} \tilde{\Phi}_{r}\left(m_{r}[\omega]-m_{s}[\eta]\right) d B_{r}^{i, s, \eta}, \quad \mathbb{Q}^{s, \eta} \text { a.s. }
\end{aligned}
$$

Proposition 4.12. We suppose the validity of Hypotheses 3.4 and 4.1. Then $\left(\mathbb{Q}^{s, \eta}\right)_{(s, \eta) \in[0, T] \times \Omega}$ solves the martingale problem associated to $(\mathcal{D}(A), A)$, see Definition 2.14. Moreover, $(\mathcal{D}(A), A)$ is a weak generator of $\left(P_{s}\right)_{s \in[0, T]}$, see Definition 2.15.

Proof. We fix $(s, \eta)$. The first item of Definition 2.14 holds by construction of $\left(\mathbb{Q}^{s, \eta}\right)_{(s, \eta) \in[0, T] \times \Omega}$, see Proposition 3.72 .

We now fix $\Phi:(t, \omega) \longmapsto \tilde{\Phi}_{t}\left(m_{t}[\omega]\right) \in \mathcal{D}(A)$ with $\tilde{\Phi} \in \mathcal{C}_{+}^{1,2}(\bar{\Lambda})$. It is not hard to see that $\tilde{\Phi}^{s, \eta}:(t, \omega) \mapsto \tilde{\Phi}_{t}\left(\omega+m_{s}[\eta]\right)$ also belongs to $\mathcal{C}_{+}^{1,2}(\bar{\Lambda})$ with

- $D \tilde{\Phi}_{t}^{s, \eta}(\omega)=D \tilde{\Phi}_{t}\left(\omega+m_{s}[\eta]\right)$,

- $\nabla_{b(\cdot, t)} \tilde{\Phi}_{t}^{s, \eta}(\omega)=\nabla_{b(\cdot, t)} \tilde{\Phi}_{t}\left(\omega+m_{s}[\eta]\right)$,

- $\nabla_{k_{i}(\cdot, t)}^{2} \tilde{\Phi}_{t}^{s, \eta}(\omega)=\nabla_{k_{i}(\cdot, t)}^{2} \tilde{\Phi}_{t}\left(\omega+m_{s}[\eta]\right), i \leq d$,

for all $(t, \omega)$. Applying Theorem 4.11 to $\tilde{\Phi}^{s, \eta}$, we obtain

$$
\begin{aligned}
\tilde{\Phi}_{t}\left(m_{t}[\cdot]\right)= & \tilde{\Phi}_{s}\left(m_{s}[\eta]\right)+\int_{s}^{t}\left(D \tilde{\Phi}_{r}+\nabla_{b(\cdot, r)} \tilde{\Phi}_{r}+\frac{1}{2} \sum_{i \leq d} \nabla_{k_{i}(\cdot, r)}^{2} \tilde{\Phi}_{r}\right)\left(m_{r}\right) d r \\
& +\sum_{i \leq d} \int_{s}^{t} \nabla_{k_{i}(\cdot, r)} \tilde{\Phi}_{r}\left(m_{r}\right) d B_{r}^{i, s, \eta}, \quad t \in[s, T]
\end{aligned}
$$


in the sense of $\mathbb{Q}^{s, \eta}$-indistinguishability. Therefore, by definition of $\Phi$ and $A$ in Definition 4.8, $\Phi-\int_{0}^{\cdot} A \Phi_{r} d r$ is on $[s, T]$ a $\left(\mathbb{Q}^{s, \eta}, \mathbb{F}^{o}\right)$-local martingale, since it is indistinguishable, from $\Phi_{s}(\eta)+\sum_{i \leq d} \int_{s}^{\cdot} \nabla_{k_{i}(\cdot, r)} \tilde{\Phi}_{r}\left(m_{r}\right) d B_{r}^{i, s, \eta}$.

Since for all $i,(t, \omega) \mapsto \nabla_{k_{i}(\cdot, r)} \tilde{\Phi}_{r}\left(m_{r}[\omega]\right)$ is assumed to have polynomial growth and $\sup _{t \in[s, T]} X_{t} \in L^{2}\left(\mathbb{Q}^{s, \eta}\right)$ then, for all $i, \sup _{t \in[s, T]}\left|\nabla_{k_{i}(\cdot, r)} \tilde{\Phi}_{r}\left(m_{r}\right)\right| \in L^{2}\left(\mathbb{Q}^{s, \eta}\right)$, therefore finally $\sum_{i \leq d} \int_{s}^{\cdot} \nabla_{k_{i}(\cdot, r)} \tilde{\Phi}_{r}\left(m_{r}\right) d B_{r}^{i, s, \eta}$ is a martingale. So $\Phi-\int_{0}^{\cdot} A \Phi_{r} d r$ is on $[s, T]$ a $\left(\mathbb{Q}^{s, \eta}, \mathbb{F}^{o}\right)$-martingale. Since this holds for any $(s, \eta)$ and $\Phi \in \mathcal{D}(A)$ then $\left(\mathbb{Q}^{s, \eta}\right)_{(s, \eta) \in[0, T] \times \Omega}$ solves the martingale problem associated to $(\mathcal{D}(A), A)$.

The second part of statement follows by Proposition 2.16.

Notation 4.13. For every $\tilde{\Phi} \in \mathcal{D}(\tilde{A})$ and $(s, \eta) \in[0, T] \times \Omega$, we denote $M[\Phi]^{s, \eta}$ the continuous $\mathbb{Q}^{s, \eta}$-martingale $\Phi-\Phi_{s}(\eta)-\int_{s}^{\cdot} A \Phi_{r} d r$, indexed by $[s, T]$.

A direct consequence of Lemma 3.14 in [2], taking into account Notation 4.13 , is the following.

Corollary 4.14. Let $\tilde{\Phi}, \tilde{\Psi} \in \mathcal{D}(A)$. Then for all $(s, \eta)$

$$
\left\langle M[\Phi]^{s, \eta}, M[\Psi]^{s, \eta}\right\rangle=\sum_{i \leq d} \int_{s}^{\cdot} \nabla_{k_{i}(\cdot, r)} \tilde{\Phi}_{r}\left(m_{r}\right) \nabla_{k_{i}(\cdot, r)} \tilde{\Psi}_{r}\left(m_{r}\right) d r
$$

with respect to $\mathbb{Q}^{s, \eta}$.

The following bilinear operator was introduced in [2] in a general pathdependent framework.

Notation 4.15. Let $\Phi, \Psi \in \mathcal{D}(A)$. We denote by $\Gamma(\Phi, \Psi)$ the process $A(\Phi \Psi)$ $\Phi A(\Psi)-\Psi A(\Phi)$. If $\Phi$ or $\Psi$ is multidimensional, then we define $\Gamma(\Phi, \Psi)$ as a vector or matrix, coordinate by coordinate. $\Gamma(\Phi, \Phi)$ will be denoted $\Gamma(\Phi)$.

$\Gamma$ can be interpreted as a path-dependent extension of the concept of carré du champ operator in the theory of Markov processes.

Proposition 4.16. For every $\tilde{\Phi}, \tilde{\Psi} \in \mathcal{D}(\tilde{A})$, we have

$$
\Gamma(\Phi, \Psi)_{t}(\omega)=\sum_{i \leq d} \nabla_{k_{i}(\cdot, t)} \tilde{\Phi}_{t} \nabla_{k_{i}(\cdot, t)} \tilde{\Phi}_{t}\left(m_{t}[\omega]\right), \forall t, \omega
$$

Proof. This directly follows from the fact that $D$ and for $\zeta, \nabla_{\zeta}$ verify the usual product rules.

\subsection{Construction of the driving martingale for the BSDE}

Notation 4.17. We will indicate by $d t \otimes d \mathbb{Q}^{s, \eta}$ the measure on $\mathcal{B}([0, T]) \otimes \mathcal{F}$ defined by $d t \otimes d \mathbb{Q}^{s, \eta}(C)=\mathbb{E}^{s, \eta}\left[\int_{s}^{T} \mathbb{1}_{C}(r, \omega) d r\right]$, and by $\mathcal{L}^{2}\left(d t \otimes d \mathbb{Q}^{s, \eta}\right)$ the space of $\mathbb{F}^{s, \eta}$-progressively measurable processes $Y$ such that $\mathbb{E}^{s, \eta}\left[\int_{s}^{T}\left|Y_{r}\right|^{2} d r\right]<\infty$. 
$\mathcal{H}_{0}^{2}\left(\mathbb{Q}^{s, \eta}\right)$ will denote the space of $\left(\mathbb{Q}^{s, \eta}, \mathbb{F}^{s, \eta}\right)$-square integrable martingales vanishing at time $s$, hence on the interval $[0, s]$ since it is $\mathbb{Q}^{s, \eta}$ a.s. deterministic on $[0, s[$, see Proposition 2.12. The elements of that space will be identified up to indistinguishability with respect to $\mathbb{Q}^{s, \eta}$.

We define $\mathcal{L}_{\text {uni }}^{2}$ as the linear space of $\mathbb{F}^{o}$-progressively measurable processes belonging to $\mathcal{L}^{2}\left(d t \otimes d \mathbb{Q}^{s, \eta}\right)$ for all $(s, \eta) \in[0, T] \times \Omega$. Let $\mathcal{N}$ be the linear subspace of $\mathcal{L}_{\text {uni }}^{2}$ constituted of elements which are equal to $0 d t \otimes d \mathbb{Q}^{s, \eta}$ a.e. for all $(s, \eta) \in[0, T] \times \Omega$. We denote $L_{\text {uni }}^{2}:=\mathcal{L}_{\text {uni }}^{2} \backslash \mathcal{N}$.

Definition 4.18. A property will be said to hold quasi surely, abbreviated by q.s. if it holds everywhere but in some $C \in \mathcal{P}$ ro $o^{o}$ such that $\mathbb{E}^{s, \eta}\left[\int_{s}^{T} \mathbb{1}_{C}(t, \omega) d t\right]=$ 0 for all $(s, \eta)$.

Notation 4.19. From now on, $m^{T}:=\left(m^{T, 1}, \cdots, m^{T, d}\right)$ will denote what we call prediction process $(t, \omega) \mapsto m_{t}[\omega](T)=\mathbb{E}^{t, \omega}\left[X_{T}\right]-\beta_{T}$, in agreement with the last statement of Remark 4.4 and Definition 4.2. For all $(s, \eta)$ we introduce the $\mathbb{R}^{d}$-valued $\mathbb{Q}^{s, \eta}$-martingale

$$
m^{T, s, \eta}:=\left(m^{T, 1, s, \eta}, \cdots, m^{T, d, s, \eta}\right):=\int_{s} k(T, r) d B_{r}^{s, \eta},
$$

indexed by $[s, T]$ (extended by convention on $[0, s]$ with the value 0 ), where we recall that $B^{s, \eta}$ is the Brownian motion introduced in Remark 4.4.

Proposition 4.20. For all $i \leq d$, we have the following.

1. $m^{T, i}$ and $\left(m^{T, i}\right)^{2}$ belong to $\mathcal{D}(A)$ with $A\left(m^{T, i}\right) \equiv 0$ and

$$
\Gamma\left(m^{T, i}\right)=\sum_{j \leq d} k_{i, j}^{2}(T, \cdot)=\left(k k^{\top}\right)_{i, i}(T, \cdot),
$$

which is bounded;

2. for every $\tilde{\Phi} \in \tilde{\mathcal{D}}(\tilde{A})$ we have $\Gamma\left(\Phi, m^{T, i}\right)_{t}(\omega)=\sum_{j \leq d} k_{i, j}(T, t) \nabla_{k_{j}(\cdot, t)} \tilde{\Phi}_{t}\left(m_{t}[\omega]\right)$, for all $t, \omega$;

3. for every $(s, \eta)$, on $[s, T], m^{T, i, s, \eta}$ is $\mathbb{Q}^{s, \eta}$-indistinguishable from $m^{T, i}-m_{s}^{T, i}(\eta)$; it belongs to $\mathcal{H}_{0}^{2}\left(\mathbb{Q}^{s, \eta}\right)$ and $\frac{d\left\langle m^{T, i, s, \eta}\right\rangle_{t}}{d t}=\sum_{j \leq d} k_{i, j}^{2}(T, t)$ is bounded $d t \otimes d \mathbb{Q}^{s, \eta}$ a.e.;

4. $m^{T, i} \in \mathcal{L}_{\text {uni }}^{2}$.

Remark 4.21. Let $1 \leq i \leq d$. For all $(t, \omega)$, by definition of canonical process, we get $m_{t}^{T, i}(\omega)=X_{T}^{i}\left(m_{t}[\omega]\right)$. So $(t, \omega) \mapsto X_{T}^{i}(\omega)$ is the $\tilde{\Phi}$ corresponding to $\Phi=m^{T, i}$ 
Proof. We fix $i \leq d$.

By Example 3.5 1. in [26] both $(t, \omega) \mapsto X_{T}^{i}(\omega),\left(X_{T}^{i}\right)^{2}(\omega)$ belong to $\mathcal{C}_{+}^{1,2}(\bar{\Lambda})$; also $D X_{T}^{i} \equiv 0, \nabla_{b(\cdot, t)} X_{T}^{i}(\omega)=b_{i}(T, t), \nabla_{k_{j}(\cdot, t)} X_{T}^{i}(\omega)=k_{i, j}(T, t)$ for all $j \leq$ $d ;(t, \omega)$ and that $\nabla_{k_{j}(\cdot, t)}^{2}\left(X_{T}^{i}\right)_{t}(\omega)=0$ for all $j \leq d,(t, \omega)$. Therefore, according to Definition 4.8, $m^{T, i}$ belongs to $\mathcal{D}(A)$ and $A\left(m^{T, i}\right) \equiv 0$. Since $\left(X_{T}^{i}\right)^{2} \in \mathcal{C}_{+}^{1,2}(\bar{\Lambda})$, then by Proposition 4.16, $\Gamma\left(m^{T, i}\right)$ is equal to $\sum_{j \leq d} k_{i, j}^{2}(T, \cdot)$ which is bounded by Hypothesis 4.1. This shows item 1.

The second statement also holds by Proposition 4.16, and the fact that $\nabla_{k_{j}(\cdot, t)} X_{T}^{i}(\omega)=k_{i, j}(T, t)$ for all $i, j \leq d$ and $(t, \omega)$.

Concerning the third statement, we have $X_{T}^{i}=m_{s}^{T, i}(\eta)+\beta_{T}^{i}+\int_{s}^{T} k_{i}(T, r) d B_{r}^{s, \eta}$ $\mathbb{Q}^{s, \eta}$ a.s. (see Remark 4.4) so that for all $t \geq s$, taking into account Notation 4.19

$$
\begin{aligned}
m_{t}^{T, i}(\omega) & =\mathbb{E}^{t, \omega}\left[X_{T}^{i}\right]-\beta_{T}^{i} \\
& =\mathbb{E}^{s, \eta}\left[X_{T}^{i} \mid \mathcal{F}_{t}^{o}\right](\omega)-\beta_{T}^{i} \quad \mathbb{Q}^{s, \eta} \text { a.s. } \\
& =m_{s}^{T, i}(\eta)+\beta_{T}^{i}-\beta_{T}^{i}+\mathbb{E}^{s, \eta}\left[\int_{s}^{T} k_{i}(T, r) d B_{r}^{s, \eta} \mid \mathcal{F}_{t}^{o}\right](\omega) \quad \mathbb{Q}^{s, \eta} \text { a.s. } \\
& =m_{s}^{T, i}(\eta)+\int_{s}^{t} k_{i}(T, r) d B_{r}^{s, \eta} \quad \mathbb{Q}^{s, \eta} \text { a.s. },
\end{aligned}
$$

where the second equality holds because $\left(\mathbb{Q}^{s, \eta}\right)$ is a path-dependent canonical class, taking into account Remark 2.5. So, according to Notation $4.19 \mathrm{~m}^{T, i, s, \eta}$ is a $\mathbb{Q}^{s, \eta}$-modification of $m^{T, i}-m_{s}^{T, i}(\eta)$. Since $m^{T, i, s, \eta}$ is $\mathbb{Q}^{s, \eta}$-a.s. continuous and $t \mapsto m_{t}^{T, i}(\omega)-m_{s}^{T, i}(\eta)$ is right-continuous for all $\omega$ (see Proposition 3.11), then those processes are indistinguishable. The quadratic variation of $m^{T, i, s, \eta}$ is $\sum_{j \leq d} \int_{s} k_{i, j}^{2}(T, r) d r$ so $\frac{d\left\langle m^{T, i, s, \eta}\right\rangle_{t}}{d t}=\sum_{j \leq d} k_{i, j}^{2}(T, t) d t \otimes d \mathbb{Q}^{s, \eta}$ a.e. is indeed bounded a.e. since $k$ is bounded. $m^{T, i, s, \eta}$ is a square integrable martingale, because its quadratic variation is bounded.

We now discuss the last statement. For all $(s, \eta), m^{T, i, s, \eta}$ is on $[s, T] \mathbb{Q}^{s, \eta_{-}}$ indistinguishable from $m^{T, i}-m_{s}^{T, i}(\eta)$ therefore $m^{T, i} \in \mathcal{L}_{\text {uni }}^{2}$ if and only if for every $(s, \eta), m^{T, i, s, \eta} \in \mathcal{L}^{2}\left(d t \otimes d \mathbb{Q}^{s, \eta}\right)$. This indeed holds, since for every $(s, \eta)$, by statement $3 ., m^{T, i, s, \eta}$ is a square integrable martingale, hence $\sup _{r \in[s, T]}\left|m_{r}^{T, i, s, \eta}\right| \in \mathcal{L}^{2}\left(\mathbb{Q}^{s, \eta}\right)$ by Doob inequality.

$r \in[s, T]$

\subsection{The semilinear path-dependent PDE and associated BSDE}

We now introduce the path-dependent PDE that interests us. We consider some $\xi, f$ verifying the following hypothesis.

\section{Hypothesis 4.22.}

1. $\xi$ is a r.v. with polynomial growth;

2. $f:([0, T] \times \Omega) \times \mathbb{R} \times \mathbb{R} \longmapsto \mathbb{R}$ is measurable with respect to $\mathcal{P}$ ro ${ }^{\circ} \otimes \mathcal{B}(\mathbb{R}) \otimes$ $\mathcal{B}(\mathbb{R})$ and such that 
(a) $f(\cdot, \cdot, 0,0)$ has polynomial growth;

(b) there exists $K>0$ such that for all $\left(t, \omega, y, y^{\prime}, z, z^{\prime}\right) \in[0, T] \times \Omega \times$ $\mathbb{R} \times \mathbb{R} \times \mathbb{R} \times \mathbb{R}$

$$
\left|f\left(t, \omega, y^{\prime}, z^{\prime}\right)-f(t, \omega, y, z)\right| \leq K\left(\left|y^{\prime}-y\right|+\left|z^{\prime}-z\right|\right) .
$$

We recall that the notion of polynomial growth has been introduced in Definition 4.6.

Remark 4.23. A direct consequence of Proposition 3.15 and the fact that $\mathbb{Q}^{s, \eta}$ is a translation of $\mathbb{P}^{s, \eta}$, is that since $\xi, f$ verify Hypothesis 4.22, then $\xi$ belongs to $\mathcal{L}^{2}\left(\mathbb{Q}^{s, \eta}\right)$ for all $(s, \eta)$ and $f(\cdot, \cdot, 0,0) \in \mathcal{L}_{\text {uni }}^{2}$. We remark that $\mathbb{Q}^{s, \eta}$ is just a translation of $\mathbb{P}^{s, \eta}$, under which $\|\omega\|_{\infty}$ admits finite moments of every order, see Proposition 3.15.

We now consider the following abstract path-dependent non linear equation.

$$
\begin{cases}A \Phi+f\left(\cdot, \cdot, \Phi, \Gamma\left(m^{T}, \Phi\right)\right)=0 & \text { on }[0, T] \times \Omega \\ \Phi_{T}=\xi & \text { on } \Omega .\end{cases}
$$

Remark 4.24. In previous equation (4.10), if $\tilde{\Phi} \in \mathcal{D}(\tilde{A})$ then the equation can also be written

$$
\left\{\begin{array}{l}
\left(D \tilde{\Phi}_{t}+\nabla_{b(\cdot, t)} \tilde{\Phi}_{t}+\frac{1}{2} \sum_{i \leq d} \nabla_{k_{i}(\cdot, t)}^{2} \tilde{\Phi}_{t}+f\left(t, \cdot, \tilde{\Phi}_{t}, k(T, t) \nabla_{k(\cdot, t)} \tilde{\Phi}_{t}\right)\right)\left(m_{t}[\omega]\right)=0 \\
\quad \text { on }[0, T] \times \Omega \\
\tilde{\Phi}_{T}(\omega)=\xi(\omega) \quad \text { on } \Omega
\end{array}\right.
$$

see Definition 4.8 and Proposition 4.20, observing that $m_{T}(\omega)=\omega$, hence $\tilde{\Phi}_{T}=\Phi_{T}$.

Notation 4.25. Equation (4.10) will be denoted PDPDE $(f, \xi)$.

Definition 4.26. A process $Y$ will be called a classical solution of $P D P D E(f, \xi)$ if it belongs to $\mathcal{D}(A)$ and if $Y$ verifies (4.10). A process $Y \in \mathcal{L}_{\text {uni }}^{2}$ will be called decoupled mild solution of PDPDE $(f, \xi)$ if there exist auxiliary processes $Z^{1}, \cdots, Z^{d} \in \mathcal{L}_{\text {uni }}^{2}$ such that for all $(s, \eta) \in[0, T] \times \Omega$ :

$$
\left\{\begin{aligned}
Y_{s}(\eta) & =P_{s}[\xi](\eta)+\int_{s}^{T} P_{s}\left[f\left(r, \cdot, Y_{r}, Z_{r}\right)\right](\eta) d r \\
\left(Y m^{T}\right)_{s}(\eta) & =P_{s}\left[\xi m_{T}^{T}\right](\eta)-\int_{s}^{T} P_{s}\left[\left(Z_{r}-m_{r}^{T} f\left(r, \cdot, Y_{r}, Z_{r}\right)\right)\right](\eta) d r
\end{aligned}\right.
$$

where $Z:=\left(Z^{1}, \cdots, Z^{d}\right)$.

The couple $(Y, Z)$ will be said to solve the identification problem $I P(f, \xi)$.

Decoupled mild solutions were introduced in path-dependent framework in [2] and in the framework of classical parabolic PDEs in [3]. 
To $P D P D E(f, \xi)$ we associate the following family of BSDEs indexed by $(s, \eta)$ and defined on the time interval $[s, T]$ :

$$
Y^{s, \eta}=\xi+\int^{T} f\left(r, \cdot, Y_{r}^{s, \eta}, \frac{d\left\langle M^{s, \eta}, m^{T, s, \eta}\right\rangle_{r}}{d r}\right) d r-\left(M_{T}^{s, \eta}-M_{\cdot}^{s, \eta}\right),
$$

in the stochastic basis $\left(\Omega, \mathcal{F}^{s, \eta}, \mathbb{F}^{s, \eta}, \mathbb{Q}^{s, \eta}\right)$, where $m^{T, s, \eta}$, which was introduced in Notation 4.19 , is the driving martingale.

Remark 4.27. Taking into account Hypothesis 4.22, Remark 4.23 and the fact that $\frac{d\left\langle m^{T, s, \eta}\right\rangle_{t}}{d t}$ is bounded $d t \otimes d \mathbb{Q}^{s, \eta}$ a.e. (see Proposition 4.20), Theorem 3.3 and Remark 3.4 in [3] applied with $\hat{M}:=m^{T, s, \eta} ; \quad V_{t} \equiv t$ imply that for every $(s, \eta)$, there exists a unique couple $\left(Y^{s, \eta}, M^{s, \eta}\right) \in \mathcal{L}^{2}\left(d t \otimes d \mathbb{Q}^{s, \eta}\right) \times \mathcal{H}_{0}^{2}\left(\mathbb{Q}^{s, \eta}\right)$ verifying (4.13) on $[s, T]$.

We state now the main results of this paper.

Theorem 4.28. Assume the validity of Hypotheses 3.4 for $\mathbb{P}, 4.1$ for $b, k$ and 4.22 for $\xi, f$.

1. PDPDE $(f, \xi)$ has a unique decoupled mild solution;

2. $I P(f, \xi)$ admits a unique solution $(Y, Z) \in \mathcal{L}_{\text {uni }}^{2} \times\left(L_{\text {uni }}^{2}\right)^{d}$. By uniqueness we mean more precisely the following: if $(Y, Z)$ and $(\bar{Y}, \bar{Z})$ are two solutions then $Y$ and $\bar{Y}$ are identical and $Z=\bar{Z}$ q.s.

3. For every $(s, \eta)$, let $\left(Y^{s, \eta}, M^{s, \eta}\right)$ be the solution of (4.13). Then, for every $(s, \eta)$, we have that, $Y^{s, \eta}$ is on $[s, T] a \mathbb{Q}^{s, \eta}$ modification of $Y$, and $Z_{t}=\frac{d\left\langle M^{s, \eta}, m^{T, s, \eta}\right\rangle_{t}}{d t} d t \otimes d \mathbb{Q}^{s, \eta}$ a.e. In particular, $Y_{s}(\eta)=Y_{s}^{s, \eta}$.

Proof. We make use of Theorem 3.19 in [2] applied with the operator $(\mathcal{D}(A), A)$ introduced in Definition 4.8 and with $\Psi:=m^{T}$.

$\left(\mathbb{Q}^{s, \eta}\right)_{(s, \eta) \in[0, T] \times \Omega}$ is a progressive path-dependent canonical class verifying Hypothesis 2.6 (see Propositions 3.10, 3.18 and Corollary 3.21) and $(\mathcal{D}(A), A)$ is weak generator of $\left(P_{s}\right)_{s \in[0, T]}$ (see Proposition 4.12) as required in Section 3.6 of [2]. $\Psi:=m^{T}$ verifies Hypothesis 3.16 in [2] thanks to Proposition 4.20, and $\xi, f$ verify Hypothesis 3.6 in [2] thanks to Hypothesis 4.22 and Remark 4.23. So Theorem 3.19 in [2] applies.

The link between decoupled mild solutions and classical solutions is the following.

\section{Proposition 4.29.}

1. Let $\Phi$ be a classical solution of $P D P D E(f, \xi)$, see Definition 4.26. Then $\left(\Phi, \Gamma\left(m^{T}, \Phi\right)\right)$ is a solution of the identification problem $I P(f, \xi)$ (see Definition 4.26) and in particular, $\Phi$ is a decoupled mild solution of PDPDE $(f, \xi)$; 
2. there is at most one classical solution of PDPDE $(f, \xi)$;

3. assume that the unique decoupled mild solution $Y$ of $P D P D E(f, \xi)$ verifies $Y \in \mathcal{D}(A)$; then $Y$ is a classical solution q.s., in the sense that $Y_{T}=\xi$ (for all $\omega$ ) and that $A(Y)=-f\left(\cdot, \cdot, Y, \Gamma\left(m^{T}, Y\right)\right)$ q.s., see Definition 4.18.

\section{Proof.}

1. Let $\Phi:(t, \omega) \longmapsto \tilde{\Phi}_{t}\left(m_{t}[\omega]\right)$ be a classical solution. First, since $\Phi$ belongs to $\mathcal{D}(A)$ then $\Phi$ and

$$
\Gamma\left(m^{T, i}, \Phi\right):(t, \omega) \longmapsto \sum_{j \leq d} k_{i, j}(T, t) \nabla_{k_{j}(\cdot, t)} \tilde{\Phi}_{t}\left(m_{t}[\omega]\right), i \leq d,
$$

have polynomial growth, see Definition 4.8. Hence thanks to Proposition 3.15 and since $\mathbb{Q}^{s, \eta}$ is a translation of $\mathbb{P}^{s, \eta}$, those processes belong to $\mathcal{L}_{\text {uni }}^{2}$.

On the other hand, let $(s, \eta) \in[0, T] \times \Omega$. By Proposition 4.12,

$$
\begin{aligned}
M[\Phi]^{s, \eta} & =\Phi-\Phi_{s}(\eta)-\int_{s} A \Phi_{r} d r \\
M\left[\Phi m^{T}\right]^{s, \eta} & =\Phi m^{T}-\Phi_{s}(\eta) m_{s}^{T}(\eta)-\int_{s} A\left(\Phi m^{T}\right)_{r} d r
\end{aligned}
$$

are $\mathbb{Q}^{s, \eta}$-martingales on $[s, T]$ vanishing at time $s$. By Definition 4.26 we have $A \Phi=-f\left(\cdot, \cdot, \Phi, \Gamma\left(m^{T}, \Phi\right)\right)$ and by Propositions 4.16, 4.20, taking into account Notation 4.15 , we have

$A\left(\Phi m^{T}\right)=\Gamma\left(\Phi, m^{T}\right)+\Phi A m^{T}+m^{T} A \Phi=\Gamma\left(\Phi, m^{T}\right)-m^{T} f\left(\cdot, \cdot, \Phi, \Gamma\left(m^{T}, \Phi\right)\right)$,

so the martingales (4.14), indexed by $[s, T]$, can be rewritten as

$\left\{\begin{aligned} M[\Phi]^{s, \eta} & =\Phi-\Phi_{s}(\eta)+\int_{s}^{\cdot} f\left(r, \cdot, \Phi_{r}, \Gamma\left(m^{T}, \Phi\right)_{r}\right) d r \\ M\left[\Phi m^{T}\right]^{s, \eta} & =\Phi m^{T}-\Phi_{s}(\eta) m_{s}^{T}(\eta)-\int_{s}^{\prime}\left(\Gamma\left(m^{T}, \Phi\right)_{r}-m_{r}^{T} f\left(r, \cdot, \Phi, \Gamma\left(m^{T}, \Phi\right)_{r}\right) d r .\right.\end{aligned}\right.$

Finally, again by Definition 4.26 we have $\Phi_{T}=\xi$, so, for any $(s, \eta)$, taking the expectations in (4.16) at $s=T$, we get

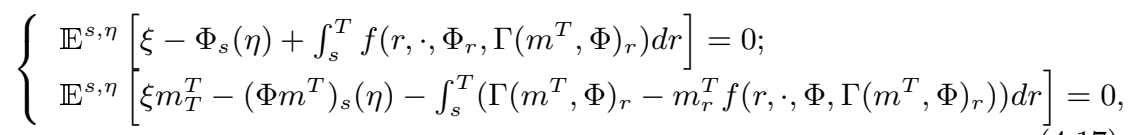

which by Fubini's theorem and Definition 2.10 yields

$$
\left\{\begin{aligned}
\Phi_{s}(\eta) & =P_{s}[\xi](\eta)+\int_{s}^{T} P_{s}\left[f\left(r, \cdot, \Phi_{r}, \Gamma\left(m^{T}, \Phi\right)_{r}\right)\right](\eta) d r \\
\left(\Phi m^{T}\right)_{s}(\eta) & =P_{s}\left[\xi m_{T}^{T}\right](\eta)-\int_{s}^{T} P_{s}\left[\Gamma\left(m^{T}, \Phi\right)_{r}-m_{r}^{T} f\left(r, \cdot, \Phi_{r}, \Gamma\left(m^{T}, \Phi\right)_{r}\right)\right](\eta) d r
\end{aligned}\right.
$$

and the first item is proved.

2. The second item follows from item 1 . and by the uniqueness of a decoupled mild solution of PDPDE $(f, \xi)$, see Theorem 4.281 . 
3. Concerning item 3. let $(Y, Z)$ be the unique solution of $I P(f, \xi)$. We first note again that the first line of (4.12) taken with $s=T$ yields $Y_{T}=\xi$.

Let us now fix some $(s, \eta) \in[0, T] \times \Omega$. The fact that $Y \in \mathcal{D}(A)$, Proposition 4.12 implies that $M^{s, \eta}[Y]=Y-Y_{s}-\int_{s} A Y_{r} d r$ is on $[s, T]$ a continuous $\mathbb{Q}^{s, \eta}$-martingale. Hence $Y$ is under $\mathbb{Q}^{s, \eta}$ a continuous semimartingale. Let us keep in mind the unique solution $\left(Y^{s, \eta}, M^{s, \eta}\right)$ of (4.13). A consequence of item 3. of Theorem 4.28 is that $Y$ admits on $[s, T]$, $Y^{s, \eta}$ as $\mathbb{Q}^{s, \eta}$ cadlag version which is a special semi-martingale verifying $Y_{t}^{s, \eta}=Y_{s}^{s, \eta}-\int_{s}^{t} f\left(r, Y_{r}, Z_{r}\right) d r+M_{t}^{s, \eta}, t \in[s, T]$. Since $Y$ is $\mathbb{Q}^{s, \eta}$ a.s. continuous, then $Y$ and $Y^{s, \eta}$ are actually $\mathbb{Q}^{s, \eta}$-indistinguishable on $[s, T]$; so the uniqueness of the decomposition of the semi-martingale $Y$, yields that $\left(\int_{s}^{*} A Y_{r} d r, M^{s, \eta}[Y]\right)$ and $\left(-\int_{s}^{*} f\left(r, Y_{r}, Z_{r}\right) d r, M^{s, \eta}\right)$ are $\mathbb{Q}^{s, \eta}$ indistinguishable on $[s, T]$. Since this holds for all $(s, \eta)$, by Definition 4.18 we have $A Y=-f(\cdot, \cdot, Y, Z)$ q.s. so we are left to show that $Z=\Gamma\left(m^{T}, Y\right)$ q.s.

For this we fix again $(s, \eta)$. By item 3. of Theorem 4.28, $\left\langle M^{s, \eta}, m^{T, s, \eta}\right\rangle=$ $\int_{s}^{*} Z_{r} d r$. By Corollary 4.14 and Propositions 4.16, 4.20, we have

$$
\left\langle M^{s, \eta}[Y], m^{T, s, \eta}\right\rangle=\int_{s} \Gamma\left(m^{T}, \Phi\right)_{r} d r .
$$

As we have remarked above, $M^{s, \eta}=M^{s, \eta}[Y]$ so $\int_{s}^{*} Z_{r} d r=\left\langle M^{s, \eta}, m^{T, s, \eta}\right\rangle=$ $\left\langle M^{s, \eta}[Y], m^{T, s, \eta}\right\rangle=\int_{s}^{\cdot} \Gamma\left(m^{T}, \Phi\right)_{r} d r$ under $\mathbb{Q}^{s, \eta}$ on $[s, T]$. Since this holds for all $(s, \eta)$, we indeed have by Definition 4.18 that $Z=\Gamma\left(m^{T}, \Phi\right)$ q.s., and the proof is complete.

\section{A Technical proofs of Section 3}

Proof of Proposition 3.10.

The fact that item 1. of Definition 2.4 holds comes from item 1. of Proposition 3.7. We now show that item 2. of Definition 2.4 holds. We fix $s \in[0, T]$. By item 2. (b) of Proposition 3.7, $\begin{aligned} & \eta \longmapsto \\ & \Omega_{s} \mathbb{P}^{s, \eta} \\ & \mathcal{P}(\Omega)\end{aligned}$ is continuous hence Borel, and by Proposition 5.3 in [4], $\mathcal{B}\left(\Omega_{s}\right)=\Omega_{s} \cap \mathcal{F}_{s}^{o}$. Since $\pi_{s}: \Omega \rightarrow \Omega_{s}$ is trivially $\left(\mathcal{F}_{s}^{o}, \Omega_{s} \cap \mathcal{F}_{s}^{o}\right)$-measurable, taking Definition 3.8 into account, it follows that $\eta \longmapsto \mathbb{P}^{s, \eta}$

$\Omega \longrightarrow \mathcal{P}(\Omega)$ is $\mathcal{F}_{s}^{o}$-measurable; so item 2. of Definition 2.4 holds.

We are left to show that its item 3. also holds. We fix $0 \leq s \leq t \leq T$. We recall that there exists a countable $\pi$-system $\Pi_{T}$ (resp. $\Pi_{t}$ ) generating $\mathcal{F}$ (resp. 
$\left.\mathcal{F}_{t}^{o}\right)$, see Notation 5.3 in [4] for instance. We fix $F \in \Pi_{T}$ and $G \in \Pi_{t}$ and we get

$$
\begin{aligned}
& \mathbb{E}^{s, \eta}\left[\mathbb{P}^{t, \zeta}(F) \mathbb{1}_{G}(\zeta)\right] \\
= & \mathbb{E}^{s, \eta}\left[\mathbb{E}^{t, \zeta}\left[\mathbb{1}_{F}(\omega)\right] \mathbb{1}_{G}(\zeta)\right] \\
= & \mathbb{E}^{s, \eta}\left[\mathbb{E}^{t, \zeta}\left[\mathbb{1}_{F}(\omega) \mathbb{1}_{G}(\zeta)\right]\right] \\
= & \mathbb{E}^{s, \eta}\left[\mathbb{E}^{t, \zeta}\left[\mathbb{1}_{F}(\omega) \mathbb{1}_{G}(\omega)\right]\right] \\
= & \mathbb{E}\left[\mathbb{E}\left[\mathbb{1}_{F} \mathbb{1}_{G} \mid \mathcal{F}_{t}^{o}\right] \mid \mathcal{F}_{s}^{o}\right](\eta), \quad \mathbb{P} \text { a.s. } \\
= & \mathbb{E}\left[\mathbb{1}_{F} \mathbb{1}_{G} \mid \mathcal{F}_{s}^{o}\right](\eta), \quad \mathbb{P} \text { a.s. } \\
= & \mathbb{E}^{s, \eta}\left[\mathbb{1}_{F} \mathbb{1}_{G}\right], \quad \mathbb{P} \text { a.s. }
\end{aligned}
$$

where the third equality holds because $G \in \mathcal{F}_{t}^{o}$ and $\mathbb{P}^{t, \zeta}\left(\omega^{t}=\zeta^{t}\right)=1$ therefore $\mathbb{1}_{G}=\mathbb{1}_{G}(\zeta) \mathbb{P}^{t, \zeta}$-a.s.; the fourth and sixth equalities hold by (3.3). Since $\Pi_{t}$ and $\Pi_{T}$ are countable, there is a set $\mathcal{N}^{c}$ of $\mathbb{P}$-full measure, such that for all $\eta$ in a set $\mathcal{N}^{c}$

$$
\mathbb{E}^{s, \eta}\left[\mathbb{P}^{t, \omega}(F) \mathbb{1}_{G}(\omega)\right]=\mathbb{E}^{s, \eta}\left[\mathbb{1}_{F} \mathbb{1}_{G}\right],
$$

for all $F \in \Pi_{T}, G \in \Pi_{t}$. By a monotone class argument, for all $\eta \in \mathcal{N}^{c}$, (A.2) holds for all $F \in \mathcal{F}, G \in \mathcal{F}_{t}^{o}$. Therefore for every $\eta \in \mathcal{N}^{c},\left(\mathbb{P}^{t, \omega}\right)_{\omega \in \Omega}$ verifies (2.1).

We will now show that $\left(\mathbb{P}^{t, \omega}\right)_{\omega \in \Omega}$ verifies $(2.1)$ for all $\eta \in \Omega$ and not just for $\eta \in \mathcal{N}^{c}$. Since $\mathcal{N}^{c}$ is of full measure, then its closure is a closed set of full measure hence is equal to $\Omega$ by Hypothesis 3.4 item 2., so $\mathcal{N}^{c}$ is dense in $\Omega$. We fix $\eta \in \Omega$, a sequence $\left(\eta_{n}\right)_{n}$ of elements of $\mathcal{N}^{c}$ converging to $\eta$, some $\Phi \in \mathcal{C}_{b}(\Omega, \mathbb{R})$ and some $\mathcal{F}_{t}^{o}$-measurable $\Psi^{t} \in \mathcal{C}_{b}(\Omega, \mathbb{R})$. For every $n$, since $\eta_{n} \in \mathcal{N}^{c}$, then $\left(\mathbb{P}^{t, \omega}\right)_{\omega \in \Omega}$ verifies (2.1) with $(s, \eta)$ replaced by $\left(s, \eta_{n}\right)$, so

$$
\mathbb{E}^{s, \eta_{n}}\left[\Phi \Psi^{t}\right]=\mathbb{E}^{s, \eta_{n}}\left[\mathbb{E}^{t, \omega}[\Phi] \Psi^{t}(\omega)\right] .
$$

$\Phi, \Psi^{t}$ are bounded continuous. By Proposition 3.7 item 2.(b), $\omega \mapsto \mathbb{P}^{t, \omega}$ is continuous; by definition of the topology on $\mathcal{P}(\Omega), \omega \mapsto \mathbb{E}^{t, \omega}[\Phi]$ is (bounded) continuous, therefore $\mathbb{E}^{t, \cdot}[\Phi] \Psi^{t}$ is bounded continuous; moreover $\Phi \Psi^{t}$ is also bounded and continuous. Since $\eta \mapsto \mathbb{P}^{s, \eta}$ is continuous, we can pass to the limit in $n$ in (A.3) and we get

$$
\mathbb{E}^{s, \eta}\left[\Phi \Psi^{t}\right]=\mathbb{E}^{s, \eta}\left[\mathbb{E}^{t, \omega}[\Phi] \Psi^{t}(\omega)\right] .
$$

(A.4) holds for all bounded continuous $\Phi$ and bounded continuous $\mathcal{F}_{t}^{o}$-measurable $\Psi^{t}$ so by the functional monotone class theorem (see Theorem 21 in [9] Chapter I), it holds for all bounded measurable $\Phi$ and bounded $\mathcal{F}_{t}^{o}$-measurable $\Psi^{t}$. Since this is true for any $\eta$ we have shown in particular that for all $\eta,\left(\mathbb{P}^{t, \omega}\right)_{\omega \in \Omega}$ verifies (2.1), i.e. item 3. of Definition 2.4.

Remark A.1. For given $s \in] 0, T], \Omega_{s}^{*}$ can be characterized as the set of bounded positive measures on $[0, s]$ not charging 0 .

The action of $\pi_{s}^{*}: \Omega_{s}^{*} \longrightarrow \Omega^{*}$ is given by $\pi_{s}^{*}(\mu): \omega \longmapsto \mu\left(\left.\omega\right|_{[0, s]}\right)$ for all $\mu \in \Omega_{s}^{*}$. 
Proof of Proposition 3.11.

We fix $\eta \in \Omega$ and $s \in[0, T$ [ and we show that $m$. [ $\eta]$ is right-continuous in $s$.

We recall that $\pi_{s} K \pi_{s}^{*} \Omega_{s}^{*}$ is dense in $\Omega_{s}$ and that on $K \pi_{s}^{*} \Omega_{s}^{*}, m_{s} \circ \pi_{s}$ coincides with the identity, see Proposition 3.7 item 2.(f). Therefore since for all $\delta \geq 0$, we clearly have $\pi_{s}^{*} \Omega_{s}^{*} \subset \pi_{s+\delta}^{*} \Omega_{s+\delta}^{*}$, so $K \pi_{s}^{*} \Omega_{s}^{*} \subset K \pi_{s+\delta}^{*} \Omega_{s+\delta}^{*}$ then for all $\epsilon \geq 0$ there exists $\eta_{\epsilon} \in K \pi_{s}^{*} \Omega_{s}^{*}$ such that

$$
\begin{aligned}
& \left\|\pi_{s}(\eta)-\pi_{s}\left(\eta_{\epsilon}\right)\right\|_{\infty} \leq \frac{\epsilon}{4 M_{o p}} ; \\
& m_{s} \pi_{s}\left(\eta_{\epsilon}\right)=\eta_{\epsilon} ; \\
& \text { for all } \delta \geq 0, m_{s+\delta} \pi_{s+\delta}\left(\eta_{\epsilon}\right)=\eta_{\epsilon} .
\end{aligned}
$$

For all $\delta \geq 0$, by Definition 3.8 we write

$$
\begin{aligned}
& m_{s+\delta}(\eta)-m_{s}(\eta) \\
= & m_{s+\delta} \pi_{s+\delta}(\eta)-m_{s} \pi_{s}(\eta) \\
= & \left(m_{s+\delta} \pi_{s+\delta}(\eta)-m_{s+\delta} \pi_{s}(\eta)\right)+\left(m_{s+\delta} \pi_{s}(\eta)-m_{s+\delta} \pi_{s}\left(\eta_{\epsilon}\right)\right) \\
& +\left(m_{s+\delta} \pi_{s}\left(\eta_{\epsilon}\right)-m_{s+\delta} \pi_{s+\delta}\left(\eta_{\epsilon}\right)\right)+\left(m_{s+\delta} \pi_{s+\delta}\left(\eta_{\epsilon}\right)-m_{s} \pi_{s}\left(\eta_{\epsilon}\right)\right) \\
& +\left(m_{s} \pi_{s}\left(\eta_{\epsilon}\right)-m_{s} \pi_{s}(\eta)\right),
\end{aligned}
$$

where the fourth term of the sum is equal to zero since by (A.5), $m_{s} \pi_{s}\left(\eta_{\epsilon}\right)=$ $\eta_{\epsilon}=m_{s+\delta} \pi_{s+\delta}\left(\eta_{\epsilon}\right)$. So we obtain

$$
\begin{aligned}
& \left\|m_{s+\delta}(\eta)-m_{s}(\eta)\right\|_{\infty} \\
\leq & \left\|m_{s+\delta} \pi_{s+\delta}(\eta)-m_{s+\delta} \pi_{s}(\eta)\right\|_{\infty}+\left\|m_{s+\delta} \pi_{s}(\eta)-m_{s+\delta} \pi_{s}\left(\eta_{\epsilon}\right)\right\|_{\infty} \\
& +\left\|m_{s+\delta} \pi_{s}\left(\eta_{\epsilon}\right)-m_{s+\delta} \pi_{s+\delta}\left(\eta_{\epsilon}\right)\right\|_{\infty}+\left\|m_{s} \pi_{s}\left(\eta_{\epsilon}\right)-m_{s} \pi_{s}(\eta)\right\|_{\infty} \\
\leq & M_{o p}\left(\left\|\pi_{s+\delta}(\eta)-\pi_{s}(\eta)\right\|_{\infty}+2\left\|\pi_{s}(\eta)-\pi_{s}\left(\eta_{\epsilon}\right)\right\|_{\infty}+\left\|\pi_{s}\left(\eta_{\epsilon}\right)-\pi_{s+\delta}\left(\eta_{\epsilon}\right)\right\|_{\infty}\right) \\
\leq & \frac{\epsilon}{2}+M_{o p}\left(\left\|\pi_{s+\delta}(\eta)-\pi_{s}(\eta)\right\|_{\infty}+\left\|\pi_{s}\left(\eta_{\epsilon}\right)-\pi_{s+\delta}\left(\eta_{\epsilon}\right)\right\|_{\infty},\right.
\end{aligned}
$$

where the second inequality holds by Proposition 3.7 item 2. (e), and the third inequality by the first line of (A.5). Since clearly, for all $\omega \in \Omega, \pi_{s+\delta}(\omega)$ tends uniformly to $\pi_{s}(\omega)$, then there exists $\delta$ small enough such that $\| \pi_{s+\delta}(\eta)-$ $\pi_{s}(\eta) \|_{\infty} \leq \frac{\epsilon}{4 M_{o p}}$ and $\left\|\pi_{s+\delta}(\eta)-\pi_{s}(\eta)\right\|_{\infty}+\left\|\pi_{s}\left(\eta_{\epsilon}\right)-\pi_{s+\delta}\left(\eta_{\epsilon}\right)\right\|_{\infty} \leq \frac{\epsilon}{4 M_{o p}}$ which combined with (A.7) gives

$$
\left\|m_{s+\delta}(\eta)-m_{s}(\eta)\right\|_{\infty} \leq \epsilon
$$

the right-continuity of $m .(\eta)$ at time $s$ is now proved.

Proof of Proposition 3.16.

We fix some $\eta_{0} \in \Omega$. It is obvious that $\operatorname{supp}\left(\mathbb{P}^{s, \eta_{0}}\right) \subset \eta_{0}^{s}+\Omega_{s}^{\perp}$. We assume that there exists an open set of $\eta_{0}^{s}+\Omega_{s}^{\perp}$ which has $\mathbb{P}^{s, \eta_{0}}$ zero measure. We will find a contradiction, and this will imply that $\operatorname{supp}\left(\mathbb{P}^{s, \eta_{0}}\right)=\eta_{0}^{s}+\Omega_{s}^{\perp} \cdot \eta_{0}^{s}+\Omega_{s}^{\perp}$ is a closed subset of $\Omega$. Suppose that there is an open subset (with respect to the induced topology) of $\eta_{0}^{s}+\Omega_{s}^{\perp}$ having zero $\left(\mathbb{P}^{s, \eta_{0}}\right)$-measure. It necessarily contains a set of type $\eta_{0}^{s}+B(\zeta, \delta) \cap \Omega_{s}^{\perp}$ where $B(\zeta, \delta)$ is the open ball (in $\Omega$ ) of 
center $\zeta \in \Omega$ and radius $\delta>0$. Then we have

$$
\begin{aligned}
& \mathbb{P}^{s, \eta_{0}}\left(\eta_{0}^{s}+B(\zeta, \delta) \cap \Omega_{s}^{\perp}\right)=0 \\
\Longleftrightarrow & \mathbb{P}^{s, \eta_{0}}\left(\omega^{s}=\eta_{0}^{s} \text { and } \pi_{s}^{\perp}(\omega) \in B(\zeta, \delta)\right)=0 \\
\Longleftrightarrow & \mathbb{P}^{s, \eta_{0}}\left(\pi_{s}^{\perp}(\omega) \in B(\zeta, \delta)=0\right. \\
\Longleftrightarrow & \mathbb{P}^{s, \eta_{0}}\left(\left(\pi_{s}^{\perp}\right)^{-1}(B(\zeta, \delta))\right)=0,
\end{aligned}
$$

where we remark that, being $\pi_{s}^{\perp}$ continuous, $\left(\pi_{s}^{\perp}\right)^{-1}(B(\zeta, \delta))$ is an open set of $\Omega$. By continuity of $m_{s}, m_{s}^{-1}\left(B\left(m_{s}\left[\eta_{0}\right], \frac{\delta}{2}\right)\right)$ is also an open set of $\Omega$.

Let $\eta \in m_{s}^{-1}\left(B\left(m_{s}\left[\eta_{0}\right], \frac{\delta}{2}\right)\right)$. Then

$$
\begin{aligned}
& \mathbb{P}^{s, \eta}\left(\left(\pi_{s}^{\perp}\right)^{-1}\left(B\left(\zeta, \frac{\delta}{2}\right)\right)\right) \\
= & \mathbb{P}^{s, \eta}\left(\omega-\omega^{s} \in B\left(\zeta, \frac{\delta}{2}\right)\right) \\
= & \mathbb{P}^{s, \eta}\left(\omega-\eta^{s} \in B\left(\zeta, \frac{\delta}{2}\right)\right) \\
= & \mathbb{P}^{s, \eta_{0}}\left(\omega-\eta^{s}+m_{s}[\eta]-m_{s}\left[\eta_{0}\right] \in B\left(\zeta, \frac{\delta}{2}\right)\right) \\
= & \mathbb{P}^{s, \eta_{0}}\left(\omega-\eta^{s}+\left(\eta^{s}+\pi_{s}^{\perp}\left(m_{s}[\eta]\right)\right)-\left(\eta_{0}^{s}+\pi_{s}^{\perp}\left(m_{s}\left[\eta_{0}\right]\right)\right) \in B\left(\zeta, \frac{\delta}{2}\right)\right) \\
= & \left.\mathbb{P}^{s, \eta_{0}}\left(\omega-\eta_{0}^{s}+\pi_{s}^{\perp}\left(m_{s}[\eta]-m_{s}\left[\eta_{0}\right]\right)\right) \in B\left(\zeta, \frac{\delta}{2}\right)\right) \\
= & \left.\mathbb{P}^{s, \eta_{0}}\left(\omega-\omega^{s}+\pi_{s}^{\perp}\left(m_{s}[\eta]-m_{s}\left[\eta_{0}\right]\right)\right) \in B\left(\zeta, \frac{\delta}{2}\right)\right) \\
= & \mathbb{P}^{s, \eta_{0}}\left(\pi_{s}^{\perp}(\omega) \in B\left(\zeta, \frac{\delta}{2}\right)-\left(m_{s}[\eta]-m_{s}\left[\eta_{0}\right]\right)\right) \\
\leq & \mathbb{P}^{s, \eta_{0}}\left(\pi_{s}^{\perp}(\omega) \in B(\zeta, \delta)\right) \\
= & 0
\end{aligned}
$$

where the third equality holds by Remark 3.13. The fourth is due to the fact that any $\omega$ can be decomposed in $\omega=\pi_{s}(\omega)+\pi_{s}^{\perp}(\omega)$ (see Notation 3.6) and that for all $\omega, m_{s}[\omega]$ and $\omega$ coincide on $[0, s]$ hence $\pi_{s}\left[m_{s}[\omega]\right]=\pi_{s}[\omega]=\omega^{s}$. The inequality holds because $\left.B\left(\zeta, \frac{\delta}{2}\right)-\left(m_{s}[\eta]-m_{s}\left[\eta_{0}\right]\right)\right) \subset B(\zeta, \delta)$ since $\| m_{s}[\eta]-$ $m_{s}\left[\eta_{0}\right] \|<\frac{\delta}{2}$, and the last equality by (A.9).

We can now consider the set $m_{s}^{-1}\left(B\left(m_{s}\left[\eta_{0}\right], \frac{\delta}{2}\right)\right) \cap\left(\pi_{s}^{\perp}\right)^{-1}(B(\zeta, \delta))$ which is open as intersection of open sets, and we compute

$$
\begin{aligned}
& \mathbb{P}\left(m_{s}^{-1}\left(B\left(m_{s}\left[\eta_{0}\right], \frac{\delta}{2}\right)\right) \cap\left(\pi_{s}^{\perp}\right)^{-1}(B(\zeta, \delta))\right) \\
= & \mathbb{E}\left[\mathbb{E}\left[\mathbb{1}_{m_{s}^{-1}\left(B\left(m_{s}\left[\eta_{0}\right], \frac{\delta}{2}\right)\right)} \mathbb{1}_{\left(\pi_{s}^{\perp}\right)^{-1}(B(\zeta, \delta))} \mid \mathcal{F}_{s}^{o}\right]\right] \\
= & \mathbb{E}\left[\mathbb{E}\left[\mathbb{1}_{\left(\pi_{s}^{\perp}\right)^{-1}(B(\zeta, \delta))} \mid \mathcal{F}_{s}^{o}\right] \mathbb{1}_{m_{s}^{-1}\left(B\left(m_{s}\left[\eta_{0}\right], \frac{\delta}{2}\right)\right)}\right] \\
= & \mathbb{E}\left[\mathbb{P}^{s, \cdot}\left(\left(\pi_{s}^{\perp}\right)^{-1}(B(\zeta, \delta))\right) \mathbb{1}_{m_{s}^{-1}\left(B\left(m_{s}\left[\eta_{0}\right], \frac{\delta}{2}\right)\right)}\right] \\
= & 0,
\end{aligned}
$$

where the second equality holds (taking into account Definition 3.8), because $m_{s}$ is $\mathcal{F}_{0}^{s}$-measurable, and the last equality holds by (A.10).

So the non-empty open set $\left.m_{s}^{-1}\left(B\left(m_{s}\left[\eta_{0}\right], \frac{\delta}{2}\right)\right) \cap \pi_{s}^{\perp}\right)^{-1}(B(\zeta, \delta))$ is of $0 \mathbb{P}$ measure, which is in contradiction with the fact that $\mathbb{P}$ is of full support as assumed in Hypothesis 3.4.

Proof of Proposition 3.18.

We will proceed showing that for every bounded r.v. $Z$,

$$
(s, \eta) \longmapsto \mathbb{E}^{s, \eta}[Z] \quad \text { is } \mathbb{F}^{o} \text {-progressively measurable. }
$$


We will make use of the functional monotone class Theorem. Let $\mathcal{C}$ be the set of r.v. of the type $e^{i \sum_{j} \lambda_{j} X_{t_{j}}^{i_{j}}}$, where $\lambda_{j}, j \leq n$ are real numbers and $i_{j}, j \leq n$ belong to $\{1, \cdots, d\}$, then $\mathcal{C}$ is stable by product and generates the $\sigma$-algebra $\mathcal{F}$. Since the set of bounded r.v. $Z$ verifying (A.12) contains all constants and is closed by uniform convergence and by monotone pointwise convergence, then by the functional monotone class Theorem (see Theorem 21 in [9] which easily extends to complex valued r.v.) it is enough to show that (A.12) holds for all $Z \in \mathcal{C}$.

We fix $n \in \mathbb{N}, i_{1}, \cdots, i_{n} \in\{1, \cdots, d\}$ and $t_{1}, \cdots, t_{n} \in[0, T]$. For any $(s, \eta)$, $\left(X_{t_{1}}^{i_{1}}, \cdots, X_{t_{n}}^{i_{n}}\right)$ is under $\mathbb{P}^{s, \eta}$ a Gaussian vector whose mean is

$$
\mu_{s, \eta}:=\left(m_{s}[\eta]^{i_{1}}\left(t_{1}\right), \cdots, m_{s}[\eta]^{i_{n}}\left(t_{n}\right)\right)
$$

and its covariance matrix $\Sigma_{s}$, where its coefficient $\Sigma_{s}(k, j)$ is equal to the $\left(i_{k}, i_{j}\right)$ th coefficient of $c^{s}\left(t_{i}, t_{j}\right)$. Therefore for all $\lambda \in \mathbb{R}^{n}$ we have

$$
\mathbb{E}^{s, \eta}\left[e^{i \sum_{j} \lambda_{j} X_{t_{j}}^{i_{j}}}\right]=e^{i\left(\lambda, \mu_{s, \eta}\right)-\frac{1}{2}\left(\lambda, \Sigma_{s} \lambda\right)} .
$$

By Corollary 3.12, m.[.] $(t)$ is $\mathbb{F}^{o}$-progressively measurable for all $t$, and by Lemma 3.17, $s \mapsto \Sigma_{s}$ is a (deterministic) continuous function. Therefore taking (A.13) into account, for all $\lambda \in \mathbb{R}^{n},(s, \eta) \longmapsto \mathbb{E}^{s, \eta}\left[e^{i \sum_{j} \lambda_{j} X_{t_{j}}^{i_{j}}}\right]$ is $\mathbb{F}^{o}$ progressively measurable, meaning that (A.12) holds with $Z \in \mathcal{C}$ and the proof is complete.

Before the proof of Proposition 3.20, we need a few technical lemmas.

Lemma A.2. Let $s \in[0, T]$. For any $n \in \mathbb{N}^{*}$ and $t_{1}, \cdots, t_{n} \in[s, T]$, the joint law of $\left(\int_{s}^{t_{j}} k_{i}\left(t_{j}, r\right) d B_{r}\right)_{j \leq n ; i \leq d}$ under $\mathbb{P}$ is equal to the joint law of $\left(X_{t_{j}}^{i}\right)_{j \leq n ; i \leq d}$ under $\mathbb{P}^{s}$.

Proof. Since both laws relate to mean-zero Gaussian vectors, it is enough to check that the covariance matrices are the same. We pick some $i_{1}, i_{2} \leq d$ and $j_{1}, j_{2} \leq n$ and through Definition 3.2, Lemma 3.17, the following calculations hold:

$$
\begin{aligned}
\mathbb{E}^{s}\left[X_{t_{j_{1}}}^{i_{1}} X_{t_{j_{2}}}^{i_{2}}\right] & =c_{i_{1}, i_{2}}^{s}\left(t_{j_{1}}, t_{j_{2}}\right) \\
& =\int_{s}^{t_{j_{1}} \wedge t_{j_{2}}} k\left(t_{j_{1}}, r\right) k\left(t_{j_{2}}, r\right) d r \\
& =\mathbb{E}\left[\int_{s}^{t_{j_{1}}} k\left(t_{j_{1}}, r\right) d B_{r} \int_{s}^{t_{j_{2}}} k\left(t_{j_{2}}, r\right) d B_{r}\right],
\end{aligned}
$$

which concludes the proof.

Corollary A.3. For all $s \in[0, T], 1 \leq i \leq d$, every finite subfamily of $\left\{\int_{s}^{t} k_{i}(t, r) d B_{r} \mid i \leq d ; t \in[0, T]\right\}$ is linearly independent in $L^{2}(\mathbb{P})$, where for all $i \leq d, k_{i}$ denotes the $i$-th raw of $k$. 
Proof. We assume that $\sum_{j \leq n, i \leq d} \lambda_{j, i} \int_{s}^{t_{j}} k_{i}\left(t_{j}, r\right) d B_{r}=0 \mathbb{P}$ a.s. where $n \in \mathbb{N}^{*} ; \lambda_{i, j} \in$ $\mathbb{R}, j \leq n, i \leq d ; t_{1}<\cdots<t_{n} \in[s, T]$.

By previous Lemma A.2, this is equivalent to having

$$
l:=\sum_{j \leq n, i \leq d} \lambda_{j, i} X_{t_{j}}^{i}=0 \quad \mathbb{P}^{s} \text { a.s. }
$$

We denote by $l_{s}$ the restriction of $l$ in (A.15) to $\Omega_{s}^{\perp}$. By (A.15), the nonempty open set $l_{s}^{-1}\left(\mathbb{R}^{*}\right)$ is of zero $\mathbb{P}^{s}$-measure, which is in contradiction with the fact that $\operatorname{supp}\left(\mathbb{P}^{s}\right)=\Omega_{s}^{\perp}$ thanks to Proposition 3.16.

Lemma A.4. For $s \in[0, T]$ we recall that the spaces $H^{s}(\mathbb{P})$ and $H(\mathbb{P})$ were introduced in Definition 3.2. Let $H^{s}(\mathbb{P})^{\perp}$ us denote the orthogonal of $H^{s}(\mathbb{P})$ in $H(\mathbb{P})$. There is an isometry $\Phi^{s}$ mapping $H^{s}(\mathbb{P})^{\perp}$ onto $H\left(\mathbb{P}^{s}\right)$ such that, for any $s$, we have the following.

1. For $t \geq s, \Phi^{s}\left(H^{s}(\mathbb{P})^{\perp} \cap H^{t}(\mathbb{P})\right)=H^{t}\left(\mathbb{P}^{s}\right)$.

2. Let $B$ be the Brownian motion whose existence is assumed in item 3. of Hypothesis 3.4. We have

$$
\Phi^{s}\left(\int_{s}^{t} k_{i}(t, r) d B_{r}\right)=X_{t}^{i}, t \geq s, i \leq d .
$$

3. $\Phi^{s}$ is the unique isometry fulfilling (A.16).

Proof. We fix $s$ and denote by $p_{s}^{\perp}$ the orthogonal projection on $H^{s}(\mathbb{P})^{\perp}$ from the space $H(\mathbb{P})$.

For all $t \geq s, 1 \leq i \leq d$, under $\mathbb{P}$ we have $X_{t}^{i}=\int_{0}^{s} k_{i}(t, r) d B_{r}+\int_{s}^{t} k_{i}(t, r) d B_{r}$, where $\int_{0}^{s} k_{i}(t, r) d B_{r} \in H^{s}(\mathbb{P})$ and $\int_{s}^{t} k_{i}(t, r) d B_{r}$ is orthogonal to ${\overline{\operatorname{Span}}\left(\left\{B_{r}^{i} \mid 1 \leq i \leq d ; r \in[0, s]\right\}\right)}^{L^{2}(\mathbb{P})}$ (which by Remark 3.5 is equal to $H^{s}(\mathbb{P})$ ), hence belongs to $H^{s}(\mathbb{P})^{\perp}$. So $p_{s}^{\perp}\left(X_{t}^{i}\right)=\int_{s}^{t} k_{i}(t, r) d B_{r}$.

Since $H(\mathbb{P})=\overline{\operatorname{Span}\left(\left\{X_{r}^{i} \mid i \leq d ; r \in[0, T]\right\}\right)} L^{2}(\mathbb{P})$ and $H^{s}(\mathbb{P})^{\perp}=p_{s}^{\perp}(H(\mathbb{P}))$ then by continuity of $p_{s}^{\perp}$,

$$
H^{s}(\mathbb{P})^{\perp}=\overline{\operatorname{Span}\left(\left\{p_{s}^{\perp}\left(X_{r}^{i}\right) \mid i \leq d ; r \in[0, T]\right\}\right)}{ }^{L^{2}(\mathbb{P})}=\bar{V}^{L^{2}(\mathbb{P})},
$$

where

$$
V=\operatorname{Span}\left\{\int_{s}^{t} k_{i}(t, r) d B_{r} \mid i \leq d ; t \in[0, T]\right\} .
$$

We start by defining $\Phi^{s}$ on $V$. First we fix $\Phi^{s}\left(\int_{s}^{t} k_{i}(t, r) d B_{r}\right)$ conformally to (A.16). Since by Corollary A.3, $\left\{\int_{s}^{t} k_{i}(t, r) d B_{r} \mid i \leq d ; t \in[0, T]\right\}$ is linearly independent, then we can extend $\Phi^{s}$ by linearity to the rest of $V$ without ambiguity. For all $t, u \geq s, i, j \leq d$, a consequence of Lemma A.2 is that 


$$
\mathbb{E}\left[\int_{s}^{t} k_{i}(t, r) d B_{r} \int_{s}^{u} k_{j}(u, r) d B_{r}\right]=\mathbb{E}^{s}\left[X_{t}^{i} X_{u}^{j}\right] .
$$

This implies that $\Phi^{s}$ preserves the scalar product, and therefore that it is an isometry from $V$ onto $\operatorname{Span}\left(\left\{X_{r}^{i} \mid i \leq d ; t \in[s, T]\right\}\right)$, as a subset of $H\left(\mathbb{P}^{s}\right)$.

By the extension theorem we can (uniquely) extend $\Phi^{s}$ to a continuous mapping from $H^{s}(\mathbb{P})^{\perp}=\overline{\operatorname{Span}\left(\left\{\int_{s}^{t} k_{i}(t, r) d B_{r} \mid i \leq d ; t \in[s, T]\right\}\right)}{ }^{2}(\mathbb{P})$ to

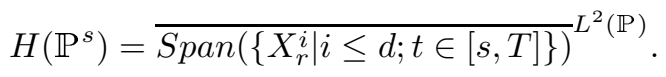

By continuity of the scalar product, $\Phi^{s}$ still preserves the scalar product, therefore the norm, and therefore is still injective. The surjectivity follows by density of $\operatorname{Span}\left(\left\{X_{t}^{i} \mid i \leq d ; t \in[s, T]\right\}\right)$ in $H\left(\mathbb{P}^{s}\right)$ and (A.16).

The proof of items 2 . are 3 . is contained in previous constructing considerations. It remains to show item 1 . We fix $t \in[s, T]$. We can argue as above and say that $H^{t}(\mathbb{P})=\overline{\operatorname{Span}\left(\left\{X_{u}^{i} \mid i \leq d ; u \in[0, t]\right\}\right)}{ }^{2}(\mathbb{P})$ and therefore that $H^{s}(\mathbb{P})^{\perp} \cap H^{t}(\mathbb{P})$ is the closure of $\operatorname{Span}\left(\left\{p_{s}^{\perp}\left(X_{u}^{i}\right) \mid i \leq d ; u \in[0, t]\right\}\right)=$ $\operatorname{Span}\left(\left\{\int_{s}^{t} k_{i}(u, r) d B_{r} \mid i \leq d ; u \in[s, t]\right\}\right)$ whose elements are mapped in $H^{t}\left(\mathbb{P}^{s}\right)$ by $\Phi^{s}$ since $\Phi^{s}\left(\int_{s}^{t} k_{i}(u, r) d B_{r}\right)=X_{u}^{i} \in H^{t}\left(\mathbb{P}^{s}\right)$ for all $u \in[s, t], i \leq d$. $H^{t}\left(\mathbb{P}^{s}\right)$ is closed and $\Phi^{s}$ is continuous, so $\Phi^{s}$ also maps

$$
\overline{\operatorname{Span}\left(\left\{\int_{s}^{t} k_{i}(u, r) d B_{r} \mid i \leq d ; u \in[s, t]\right\}\right)} L^{2}(\mathbb{P})=H^{s}(\mathbb{P})^{\perp} \cap H^{t}(\mathbb{P}),
$$

into $H^{t}\left(\mathbb{P}^{s}\right)$. Conversely, $\left(\Phi^{s}\right)^{-1}$ maps $X_{u}^{i}$ to $\int_{s}^{t} k_{i}(u, r) d B_{r} \in H^{s}(\mathbb{P})^{\perp} \cap H^{t}(\mathbb{P})$ for all $u \in[s, t], i \leq d$. By continuity of $\left(\Phi^{s}\right)^{-1}$ (the inverse of an isometry being an isometry) and since $H^{s}(\mathbb{P})^{\perp} \cap H^{t}(\mathbb{P})$ is closed, $\left(\Phi^{s}\right)^{-1}$ also maps $\overline{\operatorname{Span}\left(\left\{X_{u}^{i} \mid i \leq d ; u \in[s, t]\right\}\right)}{ }^{L^{2}(\mathbb{P})}=H^{t}\left(\mathbb{P}^{s}\right)$ into $H^{s}(\mathbb{P})^{\perp} \cap H^{t}(\mathbb{P})$. This proves item 1.

In the proof of Proposition 3.20, we will denote by $\mathcal{F}_{t}^{o, s, \eta}$, the $\sigma$-field $\mathcal{F}_{t}^{o}$ augmented with $\mathbb{P}^{s, \eta}$-null sets.

Proof of Proposition 3.20.

By Notation 3.14 we recall that under $\mathbb{P}^{s, \eta}$, the law of $X^{s, \eta}=X-m_{s}[\eta]$ is $\mathbb{P}^{s}$, where $m_{s}[\eta]$ is the deterministic function, introduced in Proposition 3.7 and Notation 3.8. Consequently $H\left(\mathbb{P}^{s, \eta}\right)=H\left(\mathbb{P}^{s}\right)$. By Lemma A.4 that space is isometric to $H^{s}(\mathbb{P})^{\perp}$. We define

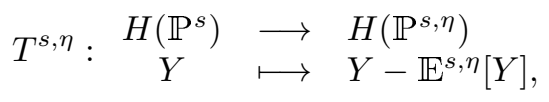

which is clearly an isometry and maps $X_{t}^{i}$ to $X_{t}^{i, s, \eta}$ for all $t \geq s, i \leq d$, hence it is easy to show

$$
T^{s, \eta}\left(H^{t}\left(\mathbb{P}^{s}\right)\right)=H^{t}\left(\mathbb{P}^{s, \eta}\right) \quad \text { for all } t .
$$


We also define

$$
\Phi^{s, \eta}:=T^{s, \eta} \circ \Phi_{s}: H^{s}(\mathbb{P})^{\perp} \longrightarrow H\left(\mathbb{P}^{s, \eta}\right),
$$

which is an isometry mapping $\int_{s}^{t} k_{i}(u, r) d B_{r}$ to $X_{t}^{i, s, \eta}$ for all $t \geq s, i \leq d$. Combining item 1. of Lemma A.4 and (A.18), it is clear that

$$
\Phi^{s, \eta}\left(H^{s}(\mathbb{P})^{\perp} \cap H^{t}(\mathbb{P})\right)=H^{t}\left(\mathbb{P}^{s, \eta}\right) \quad \text { for all } t \geq s .
$$

By Remark 3.5 item 2., $H(\mathbb{P})$ contains all the r.v. related to $B$. Moreover $B_{t}^{j}-B_{s}^{j}$ is for all $j \leq d$ and $t \geq s$ orthogonal to $\overline{\operatorname{Span}\left(\left\{B_{r}^{i} \mid i \leq d ; r \in[0, s]\right\}\right)}{ }^{2}(\mathbb{P})$ which is equal to $H^{s}(\mathbb{P})$ again by Remark 3.52 .

So for all $t \geq s, i \leq d$,

$$
B_{t}^{i}-B_{s}^{i} \in H^{s}(\mathbb{P})^{\perp} \cap H^{t}(\mathbb{P})
$$

and we denote $B_{t}^{i, s, \eta}:=\Phi^{s, \eta}\left(B_{t}^{i}-B_{s}^{i}\right)$ which by (A.20) verifies

$$
B_{t}^{i, s, \eta} \in H^{t}\left(\mathbb{P}^{s, \eta}\right) .
$$

We also denote $B^{s, \eta}:=\left(B^{1, s, \eta}, \cdots, B^{d, s, \eta}\right) . H\left(\mathbb{P}^{s, \eta}\right)$ is a Gaussian space of mean-zero r.v. so clearly, $\left(B_{t}^{s, \eta}\right)_{t \in[s, T]}$ is a mean-zero Gaussian process. Taking into account the isometry $\left(\Phi^{s, \eta}\right)^{-1}$, see considerations after (A.21), for all $s \leq$ $r, t, u, v \leq T, i, j \leq d$, we have

$$
\begin{aligned}
\mathbb{E}^{s, \eta}\left[\left(B_{v}^{i, s, \eta}-B_{u}^{i, s, \eta}\right)\left(B_{r}^{j, s, \eta}-B_{t}^{j, s, \eta}\right)\right] & =\left\langle B_{v}^{i, s, \eta}-B_{u}^{i, s, \eta}, B_{r}^{j, s, \eta}-B_{t}^{j, s, \eta}\right\rangle_{H(\mathbb{P} s, \eta)} \\
& =\left\langle B_{v}^{i}-B_{u}^{i}, B_{r}^{j}-B_{t}^{j}\right\rangle_{H(\mathbb{P})} \\
& =\mathbb{E}\left[\left(B_{v}^{i}-B_{u}^{i}\right)\left(B_{r}^{j}-B_{t}^{j}\right)\right] .
\end{aligned}
$$

In particular, under $\mathbb{P}^{s, \eta},\left(B_{t}^{s, \eta}\right)_{t \in[s, T]}$ has independent increments, and for all $s \leq t \leq u, B_{u}^{s, \eta}-B_{t}^{s, \eta}$ has variance $u-t$.

By Kolmogorov's Theorem (see Theorem 2.8 and Problem 2.10 in [19]) $\left(B_{t}^{s, \eta}\right)_{t \in[s, T]}$ admits a $\mathbb{P}^{s, \eta}$ continuous version which is a Brownian motion starting in $s$ and which we still denote $\left(B_{t}^{s, \eta}\right)_{t \in[s, T]}$. We prove below that this Brownian motion is adapted to $\left(\mathcal{F}_{t}^{o, s, \eta}\right)_{t \in[s, T]}$, that filtration being introduced just after the statement of the present Proposition 3.20. Indeed, for all $t, i \leq d$, we have by (A.22) that $B_{t}^{i, s, \eta} \in H^{t}\left(\mathbb{P}^{s, \eta}\right)$. So $B_{t}^{i, s, \eta}$ is the $L^{2}\left(\mathbb{P}^{s, \eta}\right)$ limit of linear combinations of values of $X$ at times prior to $t$, hence it is $\mathcal{F}_{t}^{o, s, \eta}$-measurable as the $L^{2}\left(\mathbb{P}^{s, \eta}\right)$ limit of $\mathcal{F}_{t}^{o, s, \eta}$-measurable r.v.

For all $i \leq d, t \geq s, \int_{s}^{t} k_{i}(t, r) d B_{r}^{s, \eta}$ is, by linearity and continuity of $\Phi^{s, \eta}$ and by construction of the Wiener integral, equal to $\Phi^{s, \eta}\left(\int_{s}^{t} k_{i}(t, r) d B_{r}\right)$. Moreover we also have $\Phi^{s, \eta}\left(\int_{s}^{t} k_{i}(t, r) d B_{r}\right)=X_{t}^{i, s, \eta}$, see the lines after (A.19). In particular $\int_{s}^{t} k_{i}(t, r) d B_{r}^{s, \eta}=X_{t}^{i, s, \eta}$ holds in $L^{2}\left(\mathbb{P}^{s, \eta}\right)$ and the first statement of Proposition 3.20 is proved.

In particular, $\left(X_{t}\right)_{t \geq s}$ is adapted to the filtration generated by $\left(B_{t}^{s, \eta}\right)_{t \in[s, T]}$ augmented with $\mathbb{P}^{s, \eta}$-null sets. Since $\left(B_{t}^{s, \eta}\right)_{t \in[s, T]}$ has been shown to be $\left(\mathcal{F}_{t}^{o, s, \eta}\right)_{t \in[s, T]}$-adapted, then $\left(\mathcal{F}_{t}^{o, s, \eta}\right)_{t \in[s, T]}$ is equal to the filtration generated 
by $\left(B_{t}^{s, \eta}\right)_{t \in[s, T]}$ augmented with $\mathbb{P}^{s, \eta}$-null sets, which is right-continuous since $\left(B_{t}^{s, \eta}\right)_{t \in[s, T]}$ is a Brownian Motion, see Proposition 7.7 in [19].

$\left(\mathcal{F}_{t}^{o, s, \eta}\right)_{t \in[s, T]}$ is therefore right-continuous. In particular, for all $t \geq s$,

$$
\mathcal{F}_{t}^{s, \eta}=\bigcap_{\epsilon>0} \mathcal{F}_{t+\epsilon}^{o, s, \eta}=\mathcal{F}_{t}^{o, s, \eta} .
$$

This concludes the "moreover" statement of Proposition 3.20.

ACKNOWLEDGEMENTS. The work of the second named author was partially supported by a public grant as part of the Investissement d'avenir project, reference ANR-11-LABX-0056-LMH, LabEx $L M H$, in a joint call with Gaspard Monge Program for optimization, operations research and their interactions with data sciences.

\section{References}

[1] C. D. Aliprantis and K. C. Border. Infinite-dimensional analysis. SpringerVerlag, Berlin, second edition, 1999. A hitchhiker's guide.

[2] A. Barrasso and F. Russo. Decoupled mild solutions of path-dependent PDEs and Integro PDEs represented by BSDEs driven by cadlag martingales. Potential Analysis. To appear.

[3] A. Barrasso and F. Russo. Martingale driven BSDEs, PDEs and other related deterministic problems. 2017. Preprint, hal-01566883.

[4] A. Barrasso and F. Russo. Path-dependent Martingale Problems and Additive Functionals. Stochastics and Dynamics, 19 no 1, 2019. Preprint, hal-01775200.

[5] J. Bion-Nadal. Dynamic risk reasures and path-dependent second order PDEs. Stochastics of Environmental and Financial Economics, 138:147178, 2016.

[6] V. I. Bogachev. Gaussian measures, volume 62 of Mathematical Surveys and Monographs. American Mathematical Society, Providence, RI, 1998.

[7] A. Cosso and F. Russo. Strong-viscosity solutions: semilinear parabolic PDEs and path-dependent PDEs. To appear: Osaka Journal of Mathematics. Preprint HAL-01145301, 2015.

[8] A. Cosso and F. Russo. Functional Itô versus Banach space stochastic calculus and strict solutions of semilinear path-dependent equations. Infin. Dimens. Anal. Quantum Probab. Relat. Top., 19(4):1650024, 44, 2016.

[9] C. Dellacherie and P.-A. Meyer. Probabilités et potentiel, volume A. Hermann, Paris, 1975. Chapitres I à IV. 
[10] C. Dellacherie and P.-A. Meyer. Probabilités et potentiel. Chapitres XIIXVI. Publications de l'Institut de Mathématiques de l'Université de Strasbourg [Publications of the Mathematical Institute of the University of Strasbourg], XIX. Hermann, Paris, second edition, 1987. Théorie des processus de Markov. [Theory of Markov processes].

[11] C. Di Girolami and F. Russo. Infinite dimensional stochastic calculus via regularization and applications. Preprint HAL-INRIA, inria-00473947 version 1, (Unpublished), 2010.

[12] B. Dupire. Functional Itô calculus. Portfolio Research Paper, Bloomberg, 2009.

[13] I. Ekren, N. Touzi, and J. Zhang. Viscosity solutions of fully nonlinear parabolic path dependent PDEs: Part I. To appear in Annals of Probability, 2013.

[14] S. N. Ethier and T. G. Kurtz. Markov processes. Wiley Series in Probability and Mathematical Statistics: Probability and Mathematical Statistics. John Wiley \& Sons, Inc., New York, 1986. Characterization and convergence.

[15] F. Flandoli and G. Zanco. An infinite-dimensional approach to pathdependent Kolmogorov equations. Ann. Probab., 44(4):2643-2693, 2016.

[16] M. Fuhrman, F. Masiero, and G. Tessitore. Stochastic equations with delay: optimal control via BSDEs and regular solutions of Hamilton-JacobiBellman equations. SIAM J. Control Optim., 48(7):4624-4651, 2010.

[17] T. Hida. Canonical representations of Gaussian processes and their applications. Mem. Coll. Sci. Univ. Kyoto. Ser. A. Math., 33:109-155, 1960/1961.

[18] J. Jacod and A. N. Shiryaev. Limit theorems for stochastic processes, volume 288 of Grundlehren der Mathematischen Wissenschaften [Fundamental Principles of Mathematical Sciences]. Springer-Verlag, Berlin, second edition, 2003.

[19] I. Karatzas and S. E. Shreve. Brownian motion and stochastic calculus, volume 113 of Graduate Texts in Mathematics. Springer-Verlag, New York, second edition, 1991.

[20] T. LaGatta. Continuous disintegrations of Gaussian processes. Theory Probab. Appl., 57(1):151-162, 2013.

[21] D. Leão, A. Ohashi, and A. B. Simas. A weak version of path-dependent functional Itô calculus. Ann. Probab., 46(6):3399-3441, 2018.

[22] É. Pardoux and S. Peng. Backward stochastic differential equations and quasilinear parabolic partial differential equations. In Stochastic partial differential equations and their applications (Charlotte, NC, 1991), volume 
176 of Lecture Notes in Control and Inform. Sci., pages 200-217. Springer, Berlin, 1992.

[23] S. Peng and F. Wang. BSDE, path-dependent PDE and nonlinear Feynman-Kac formula. Science China Mathematics, 59(1):19-36, 2016.

[24] T. Sottinen and L. Viitasaari. Prediction law of fractional Brownian motion. Statist. Probab. Lett., 129:155-166, 2017.

[25] D. W. Stroock and S. R. S. Varadhan. Multidimensional diffusion processes. Classics in Mathematics. Springer-Verlag, Berlin, 2006. Reprint of the 1997 edition.

[26] F. Viens and J. Zhang. A Martingale Approach for Fractional Brownian Motions and Related Path Dependent PDEs. ArXiv e-prints, December 2017. 